\title{
A FIRST EVER DETAILED ECOLOGICAL EXPLORATION OF THE WESTERN HIMALAYAN FORESTS OF SUDHAN GALI AND GANGA SUMMIT, AZAD JAMMU AND KASHMIR, PAKISTAN
}

\author{
KHAN, A. M..$^{1,2^{*}}$ - QURESHI, R. ${ }^{2}-$ SAQIB, Z. ${ }^{3}-$ MUNIR, M. ${ }^{4}-$ SHAHEEN, H. $^{5}-$ HABIB, ${ }^{5}{ }^{5}-$ DAR, \\ M. E. U. I. ${ }^{5}$ - FATIMAH, H. ${ }^{6}-$ AFZA, R. ${ }^{7}-$ HUSSAIN, M. A. ${ }^{8}$ \\ ${ }^{1}$ Department of Botany, Govt. Hashmat Ali Islamia Degree College, Rawalpindi, Pakistan \\ ${ }^{2}$ Department of Botany, Pir Mehr Ali Shah Arid Agriculture University, Rawalpindi, Pakistan \\ ${ }^{3}$ Department of Environmental Science, International Islamic University, Islamabad, Pakistan \\ ${ }^{4}$ Department of Botany, University of Animal \& Plant Sciences, Ravi Campus, Pattoki, Punjab, \\ Pakistan
}

${ }^{5}$ Department of Botany, University of Azad Jammu \& Kashmir, Muzaffarabad, Pakistan

${ }^{6}$ Department of Biology \& Environmental Science, Allama Iqbal Open University, $\mathrm{H}-8$ Islamabad, Pakistan

${ }^{7}$ Department of Botany, Hazara University Mansehra, Khyber Pakhtunkhwa, Pakistan

${ }^{8}$ Department of Biotechnology, Mirpur University of Science \& Technology, 10250 Mirpur, AJK, Pakistan

*Corresponding author

e-mail: arshadbotanist@gmail.com; phone: +92-33-3521-7235

(Received 22 ${ }^{\text {nd }}$ Jul 2019; accepted $16^{\text {th }}$ Oct 2019)

\begin{abstract}
The baseline ecological exploratory studies of floristically rich areas like the western Himalaya are very important to determine the diversity variations. Based on research gaps detected in ecological literature of the western Himalayan forests of Sudhan Gali and Ganga summit, the study area is revisited. Plots data was collected during 2016, and advanced multivariate statistical tools were used to analyze it. Some of multivariate tools including permutation test, indicator species analysis and variation partitioning (partial CCA) were used for the first time in the area. The results of hierarchical clustering and permutation test revealed that there were four ecologically meaningful vegetation groups (1. BerberisMicromeria-Desmodium (BMD), 2. Pinus-Rubus-Persicaria (PRP)，3. Viburnum-Abies-Dryopteris (VAD) and 4. Sibbaldia-Thymus-Bistorta (STB) associations) in the area. A significant ( $\leq \leq 0.05$ ) pairwise compositional difference of all the detected plant communities was observed. The results of canonical correspondence analysis (net effect) depicted that all the studied environmental variables were significantly important, and explains about $73.1 \%$ variations in the species data. The higher $(r>0.9)$ species-environment pseudo-canonical correlations values for the first four CCA-axes revealed that the selected explanatory variables were determinants of the response (species) data variations, and the high values for the CCA axes 2 to 4 indicated that there was no single dominant environmental gradient in this part of the western Himalaya. The highest $\beta$ diversity value of Himalayan alpine scrubland at the Ganga summit depicted that even a minor variation in environment affects the high altitude vegetation more rapidly than the vegetation of low elevation areas. The vegetation of the study area is under continuous deterioration, and this exploratory work can be used as a baseline study for the future management, sustainable use, and conservation programs in the area
\end{abstract}

Keywords: canonical correspondence analysis, biodiversity, indicator species analysis, Monte Carlo permutation test, variation partitioning, vegetation types, phytosociology 


\section{Introduction}

The ecological studies of the forests are necessary to determine the state of biodiversity pattern, vegetation structure, and species compositional dynamics over time and space. These baseline ecological studies further help in development of forest management, sustainable use of forest resources, and conservation strategies. Therefore, identification of environmental requirements of especially vulnerable plant species of any region is important and can be further used to model and identify suitable habitats. Being placed at the bottom of trophic level, plants not only serve as baseline resources for the biotic components of the higher levels, but also effectively remained engaged in maintaining the biodiversity of an area and mitigate the adverse impacts of the threatening climate changes (Khan et al., 2018a, 2019). The interactions of the various environmental factors like climate, soil, topography and anthropogenic activities strongly influence and control the vegetation structure, types, species composition and their distribution patterns (Salama et al., 2016). Therefore, the impacts of these changes on the vegetation of a given area should be studied on regular basis including speciesenvironment relationships, both on temporal and spatial scale (Akhlaq et al., 2018; Ilyas et al., 2018). The Himalayan ecosystem in general and the western Himalayan area in particular is a floristically rich area. The western Himalayan region of Pakistan is also the most fragile, comprised of about $67 \%$ of total forest cover of the country, and under serious threat of climate changes amplified by overpopulation (Qamer et al., 2016; Tewari et al., 2017). Therefore, the area need immediate attention of the ecologists to understand the biodiversity and its distribution pattern (Rahman et al., 2017; Haq, 2018; Saima et al., 2018; Kamran et al., 2019).

Community ecology deals with the documentation of number and composition of plant species/assemblages within a geographic unit. Ecologists record multivariate field data of plots, releves or quadrats, and perform multivariate analyses to test their hypotheses. Classification of vegetation types, structure and composition of any area can be evaluated more easily and efficiently by using the tools of computer modelling and multivariate statistics (viz. hierarchical clustering, indicator species analysis, ordination etc.) for the last 04 decade (Legendre and Legendre, 1998; Khan et al., 2015; Abdel Khalik et al., 2017; Bürzle et al., 2017; Devetter et al., 2017; Chandra et al., 2018; Salama et al., 2018; Zakaria et al., 2019). Constrained ordination models or direct gradient analyses techniques including unimodal model like canonical correspondence analysis (CCA) and linear model like redundancy analysis (RDA) are the most popular ones in this regard. CCA was developed by Ter Braak (1986) by arranging a marriage between multiple regression and correspondence analysis (CA). It can be used to assess the speciesenvironment relationships and its strength. In CCA, statistical significance can be evaluated by using the permutation test for overall the model, CCA axes, and explanatory variables. The CCA ordination diagram can be interpreted by following the centroid rule; species/response points are located at the centroid (weighted average) of the samples points where it is distributed. Similarly, all the samples having a set of particular species are scattered around the same species. The other information in such ordination biplot/triplot includes the importance of explanatory variables (arrow lengths), correlation of the variables to the CCA axes (direction of the arrows), correlation amongst the variables (angle amongst the arrows), ranking of the species response (optimum) and samples values for the environmental variable (perpendiculars drawn from species/samples points to environmental arrows). CCA calculates species-environment correlation which is actually a correlation between two types of site scores i.e. linear 
combinations of the environmental variables (LC's) and the weighted averages of the species scores (WA's) (Ter Braak, 1986; Legendre and Legendre, 1998).

Sudhan Gali and Ganga hills remained the focal site for vegetation analyses in the past (Table 6) due to its complex pattern of topography, extended elevation range, thick woody vegetation cover, species richness and scenic beauty of the area. However the complex interactions between the local vegetation and its environment, classification and ordination by using the multivariate tools are not documented. First ecological exploration (vegetation structure, composition and phenology) in the study area was conducted by Khan (2001), but environmental influence was not tested by using any multivariate statistical analysis. The same is true about the subsequent study conducted by Malik et al. (2007). The use of multivariate statistical methods to vegetation data of the area was started by Siddiqui et al. (2010a, b) followed by Siddiqui et al. (2014) and Shaheen et al. (2016, 2017, 2018). An unconstrained linear ordination model (PCA) was used in all of these earlier studies, therefore the real contribution of the studied predictors was missing. A total of two separate studies conducted by Shaheen et al. (2011c, 2012) attempted to use constrained unimodal ordination (CCA) to determine the species-environment relationship, but error like model overfitting was detected. The assessment of edaphic predictor's contribution was also missing in all of these earlier studies. In Pakistan, the major issue was the modest understanding about the use of advanced multivariate statistical methods in ecology, which is now improving over time. By considering this, many geographical areas of Pakistan need revisit and attention of ecological workers. It was hypothesized in every earlier vegetation study that the local environment is significantly controlling the species distribution pattern in the area but until this study, the following un-answered questions were thoroughly investigated for the first time to achieve the set objectives. (1) What are the number of biological meaningful vegetation groups, their composition and indicator species? (2) What about the species-environment relationships and contribution of the studied environment? (3) How diversity and richness in the area is related to the studied environment? (4) How the groups of the studied variables are important (partial CCA/variations partitioning) in the area? Therefore, the findings of this study will serve as the first detailed document and help in future biodiversity conservation and management projects in the region.

\section{Materials and methods}

\section{Study area}

Sudhan Gali and Ganga hills are part of the western Himalayan mountainous area in the district Bagh, Azad Jammu and Kashmir, Pakistan. It is located toward the north and about $20 \mathrm{~km}$ road distance from the Bagh city. It lies between $34^{\circ} 02^{\prime}$ to $34^{\circ} 04^{\prime} \mathrm{N}$ latitude and $73^{\circ} 44^{\prime}$ to $73^{\circ} 45^{\prime} \mathrm{E}$ longitude, and elevation ranges from 1195 to $3004 \mathrm{~m}$ above sea level (m.). It is bounded by the Hattian Bala and Muzaffarabad districts in the north, Haveli district towards the east, Poonch in the south, and Murree and Hazara hills in the west. The total land area is $770 \mathrm{~km}^{2}$, and human population of 0.434 million in 2013 with an annual growth rate of 2\% (Shaheen et al., 2011a; Anonymous, 2015) (Fig. 1).

\section{Climate, soil, and natural vegetation}

The climate of the study area is humid, sub-continental highland type, and during the last ten years (2007-2016), the mean monthly maximum temperature in June varied from 
20-26 ${ }^{\circ} \mathrm{C}$, whereas the winter temperature mostly drops to or below freezing point. Similarly, the mean annual minimum temperature varied from $6.31-13.19{ }^{\circ} \mathrm{C}$, and the mean maximum temperature varied from $20.36-27^{\circ} \mathrm{C}$. The mean annual rainfall of the same period varied from $463 \mathrm{~mm}$ in the year 2009 to $2242 \mathrm{~mm}$ in the year 2015, whereas only two months including July and August received the maximum ( $>50 \%$ ) during monsoon showers. During the year 2016, the mean annual minimum temperature, maximum temperature, and precipitation remained $7.91{ }^{\circ} \mathrm{C}, 22.45^{\circ} \mathrm{C}$, and $1798 \mathrm{~mm}$ respectively (PMD, 2018). Snowfall frequently occurs from December to February and mountains remain covered with snow until the end of April each year (Waseem et al., 2006; Saeeda and Zakir, 2012). Temperature and precipitation data (2007-2016 = 10 years) of the study area was acquired from the United States National Centers for Environmental Prediction (US-NCEP) Climate Forecast System Reanalysis (CFSR) by using the climate engine (https://app.climateengine.org/). The temperature data source was CFSv2 $19200 \mathrm{~m}$ (1/5-deg) daily reanalysis dataset (NOAA). The wind blows from west to east during the day time, and from south-east to north during the night. The wind velocity was higher in the afternoon as compared to morning time. There is no river in the study area, although a big seasonal stream "Nala Mahl" flows through the area.

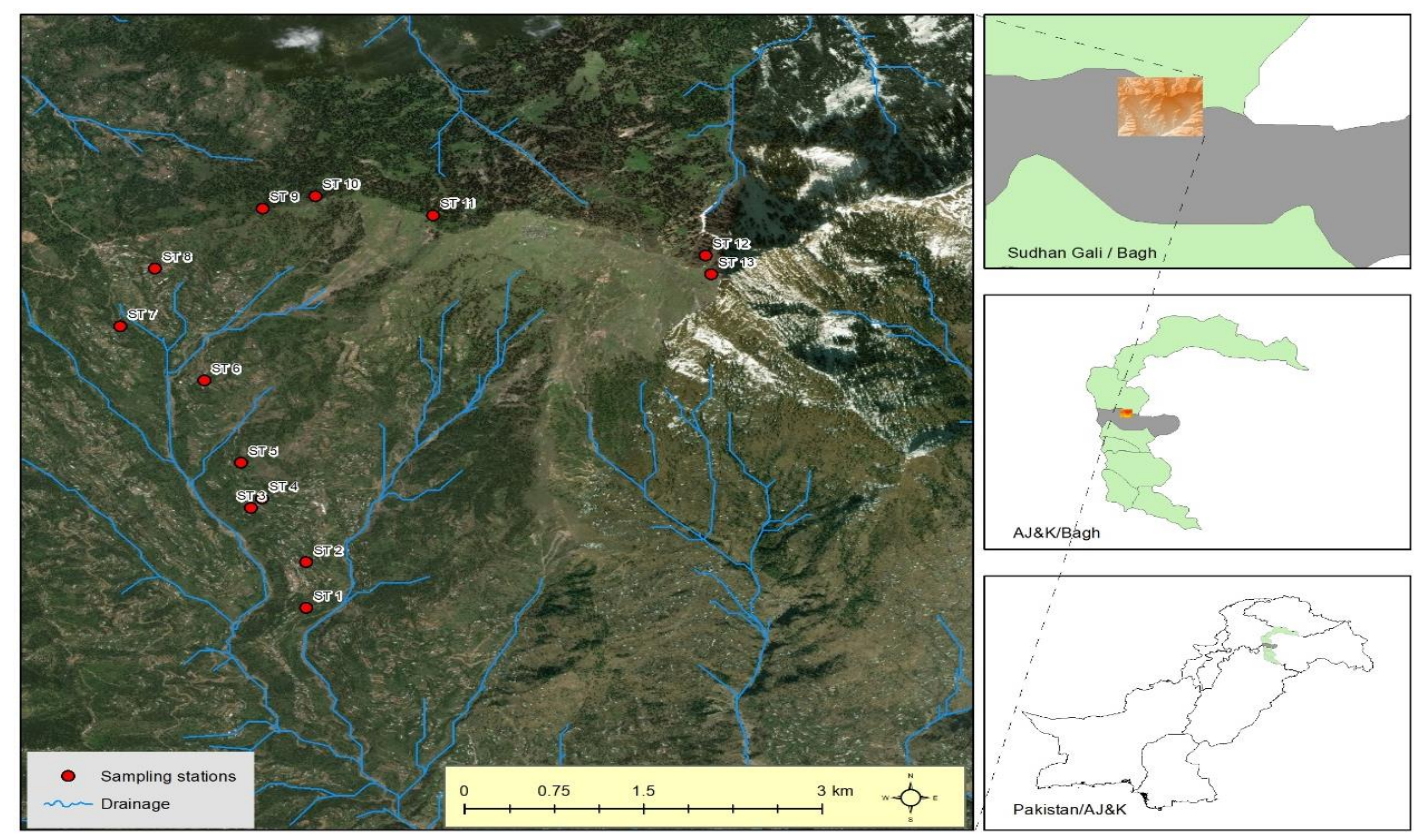

Figure 1. A GIS map of the Sudhan Gali and Ganga hills showing the distribution of 13 sampled stations in the study area

The soil of the study area is varied from clay loam to sandy loam in texture, and from yellowish brown to dark brown or to black in color. The bedrock is comprised of two types of rocks; light grey to black coloured Dogra slates of early Cambrian area (comprised of slates with quartz and feldspar, phyllites, and trilobites) and sedimentary Murree formations of the Miocene (shale and red clastic sand or sandstone) (Khan, 2001).

The natural vegetation of the area is mainly comprised of three eco-zones. These include humid sub-tropical Chir-pine, moist temperate blue-pine, and Himalayan subalpine coniferous zones. The important tree element of the natural vegetation includes 
Pinus roxburghii, P. wallichiana, and Abies pindrow. Similarly, the shrub layer is comprised of Berberis lycium, Rubus niveus, Rosa moschata, Indigofera heterantha, and Viburnum grandiflorum. The important herbaceous elements includes Micromeria biflora, Galium elegans, Veronica laxa, Oenothera rosea, Rumex nepalensis, $R$. dentatus, G. wallichianum, Achillea millefolium, Trifolium repens, Sibbaldia procumbens, Thymus linearis, and Persicaria amplexicaulis.

\section{Vegetation (plot) data collection}

A stratified random method of vegetation sampling by using plots was adopted to study the vegetation types and intensity of anthropogenic disturbance in the area. The study area was surveyed during the growing season (Monsoon) of the years 2016. A total of 13 sampling stations were randomly selected along the altitudinal gradient. At each station, three sub-stations were again randomly selected, and based on minimal area method, at each of 39 sub-station, 3 plots were randomly laid and studied to record the data of tree layer (size: $30 \times 30 \mathrm{~m}^{2}$ ). Nested plots for shrub layer (size: $5 \times 5 \mathrm{~m}^{2}$ ) and herb layers (size: $1 \times 1 \mathrm{~m}^{2}$ ) were also studied. The absolute values of density, frequency and cover of each plant species were recorded, averaged, relative values were calculated, followed by calculation of importance values index (IVI) of each species (Curtis and McIntosh, 1950; Cox, 1967; Khan et al., 2018b, 2019). First three alphabets of each of generic and specific binomials separated by a dot of each plant species were created and used in all multivariate analyses.

Geographic coordinates (locations) of each studied plot were recorded by using Garmin, 76CSx handheld GPS device and topographic variables say altitude, slope, and slope aspect were calculated by using $30 \mathrm{~m}$ digital elevation model (URL: https://asterweb.jpl.nasa.gov/gdem.asp) of the study area. The ArcGIS (ver. 10.3) was used to extract the values. Cos-transformation of the slope aspect (CTA) values was performed prior to use in the multivariate analysis. The number of stumps and herbivore dungs were also counted to measure and test the deforestation and grazing pressure respectively in each plot (Khan et al., 2019).

The collected plant specimens were pressed, dried and mounted on standard sized herbarium sheets and identified by using the available local and regional taxonomic literature (Stewart, 1972; Nasir and Ali, 1971-1995; Ali and Qaiser, 1995-2009; Nakaike and Malik, 1992; EFLORAS, 2012; TROPICOS, 2012; Khan et al., 2016), cross-matched with herbaria collections at the University of Azad Jammu and Kashmir, Muzaffarabad and deposited in the same herbarium for future references and record.

For edaphic contribution, soil samples (about $500 \mathrm{~g}$ ) were collected from each plot and topsoil layer (about $30 \mathrm{~cm}$ depth), and a composite mixture (about $1.5 \mathrm{~kg}$ ) was prepared for each sub-station. A total of six physicochemical parameters of the soil samples were studied at the Soil and Water testing Laboratory Rawalpindi, Pakistan. The soil samples were dried at room temperature followed by sieving ( $2 \mathrm{~mm}$ sieve) and storage. The soil $\mathrm{pH}$ was assessed by using 1:5 soil-water suspension and $\mathrm{pH}$ meter (Schofield and Taylor, 1955), whereas electrical conductance $\left(\mathrm{mS} \mathrm{cm}^{-1}\right)$ ability of the soil samples was tested as conveyed by Richards (1954). Soil organic matter (\%) was calculated by using the standardized solutions of $\mathrm{FeSO}_{4}$ and $\mathrm{K}_{2} \mathrm{Cr}_{2} \mathrm{O}_{7}$ as communicated by Nelson and Sommer (1982). Available phosphorus (ppm) in soil was determined by using $\mathrm{NaHCO}_{3}$ solution as extracting agent (Olsen et al., 1954). Similarly, available potassium (ppm) in soil was calculated by using the standardized solutions of $\mathrm{CH}_{3} \mathrm{COONH}_{4}$ and $\mathrm{KCl}$ (Richards, 1954). The nitrate-N (mg/kg) of soil samples was 
measured by using 1:5 soil-water suspension, and LAQUA twin Nitrate Ion meter (Othman et al., 2014).

\section{Statistical analyses}

The decision about the number of biologically meaningful plant associations in the areas was made on the basis of Monte Carlo permutation test results. A total of two to six (2-6) possible groups were tested, and the mean p-value and the number of significant indicator species were observed. A criterion of minimized p-value and/or maximized significant species was used to decide the number of plant association (Khan et al., 2018b, 2019). For ecological classification of the studied samples, two-way agglomerative hierarchical clustering (distance "Euclidean"; linkage "Ward") dendrogram of the studied vegetation samples (sub-stations) and their allied plant species was developed. Multi-response permutation procedure (MRPP) test was used to identify the pairwise compositional difference of the identified vegetation groups. Indicator species analysis (Dufrêne and Legendre, 1997) was used to identify the indicators of each group. The different diversity measure including species richness (Margalef, 1958), Shannon's diversity (Shannon and Weaver, 1949), Simpson's reciprocal diversity index (1/D; Magurran, 1988), and evenness (Pielou, 1975) were calculated for each plant association. Unimodal ordination models including detrended correspondence analysis (DCA) and canonical correspondence analysis (CCA) were used to seek the beta-diversity variations and species-environment relationships respectively. The significance of the studied variables was tested by using both simple (marginal) and conditional (net) terms effects via permutation test available in Canoco software (Ter Braak and Smilauer, 2012). Similarly, the significance of the constrained axes and pairwise comparasion of the three groups of variables (viz. topographic, edaphic and anthropogenic) was tested by using variation partitioning (partial CCA) method to seek their contribution towards the species distribution pattern in the study area (Khan et al., 2019).

\section{Results}

\section{Taxonomic and life form classification}

A total of 90 plant species of the vascular plants were recorded in the studied samples (39 sub-stations $\times 9$ plots at each sub-station $=351$ total plots studied $)$, and were allied to 78 genera and 41 families. The majority of plant species $(81,90.01 \%)$ were belonged to the angiosperms and found distributed in 71 genera and 38 families. The gymnosperms were represented by a single Pinaceae family, further comprising of 3 genera and 4 coniferous species (4.45\%) in the area. Pteridophytes were represented by 2 families, 4 genera and 5 species (Table 1 ).

Life form classification of the same 90 plant species depicted that there were 14 $(15.56 \%)$ megaphanerophytes, 9 (10\%) nanophanerophytes, $30 \quad(33.3 \%)$ hemicryptophytes, $21(23.3 \%)$ therophytes, 5 (5.56\%) chamaephytes, $10(11.1 \%)$ geophytes and $1(1.1 \%)$ liana species in the area (Table 4).

\section{Biologically meaningful vegetation associations in the area}

The first-ever results of Monte Carlo permutation testing from the area for two to six (2-6) possible vegetation groups depicted that the minimum mean p-value of 0.098 , and 
maximum number of significant indicator species of 59 were recorded at the $4^{\text {th }}$ level of division. Therefore, this study recognized a total of four (4) biologically meaningful (significant) vegetation clusters in the study area (Fig. 2). The two-way agglomerative hierarchical clustering dendrogram of the vegetation associations (grouping pattern of the 39 studied samples) and their allied plant species (90) is presented in Figure 3. A pairwise comparasion of the detected plant association was made by using MRPP test for further confirmation. The results showed that all the 6 pairwise comparisons of the 4 groups were statistically significant $(\mathrm{p}<0.001)$, therefore depicting a reasonable species compositional differences amongst the detected groups. The higher negative T-values were observed for all the pairwise comparisons, and we know that a more negative $\mathrm{T}$ value represent stronger separation of the compared plant communities. On the basis of three leading diagnostic plant species of each group (detected by using indicator species analysis, ISA), the vegetation associations were renamed as BMD (group 1), PRP (group 2), VAD (group 3) and STB (group 4). The maximum compositional separation (T-value: -17.26; p-value < 0.01) was observed between BMD and VAD associations because the former one belong to sub-tropical and the latter one to sub-alpine zone in the area. This analysis also gave A-values (within-group agreement) in the range of 0.2 to 0.37 for overall and all the 6 pairwise comparisons of the plant communities. This proves that within groups heterogeneity equals expectation by chance. The maximum A-value can be $A_{\max }=1$, which means that all items/samples within the group are identical. Conversely, $\mathrm{A}=0$ mean that within groups heterogeneity equals expectation by chance and if the A-value is < 0 , it mean that within groups heterogeneity is higher than expected by chance (Table 2 ).

Table 1. A table showing the summary of taxonomic classification of the plant species

\begin{tabular}{c|c|c|c}
\hline Phyto-taxa & Families (\%) & Genera (\%) & Species (\%) \\
\hline Pteridophytes & $2(4.88 \%)$ & $4(5.13 \%)$ & $5(5.56 \%)$ \\
Gymnosperms & $1(2.44 \%)$ & $3(3.85 \%)$ & $4(4.45 \%)$ \\
Monocots & $3(7.32 \%)$ & $11(14.11 \%)$ & $12(13.34 \%)$ \\
Dicots & $35(85.37 \%)$ & $60(76.93 \%)$ & $69(76.67 \%)$ \\
Total & $41(100 \%)$ & $78(100 \%)$ & $90(100 \%)$ \\
\hline
\end{tabular}

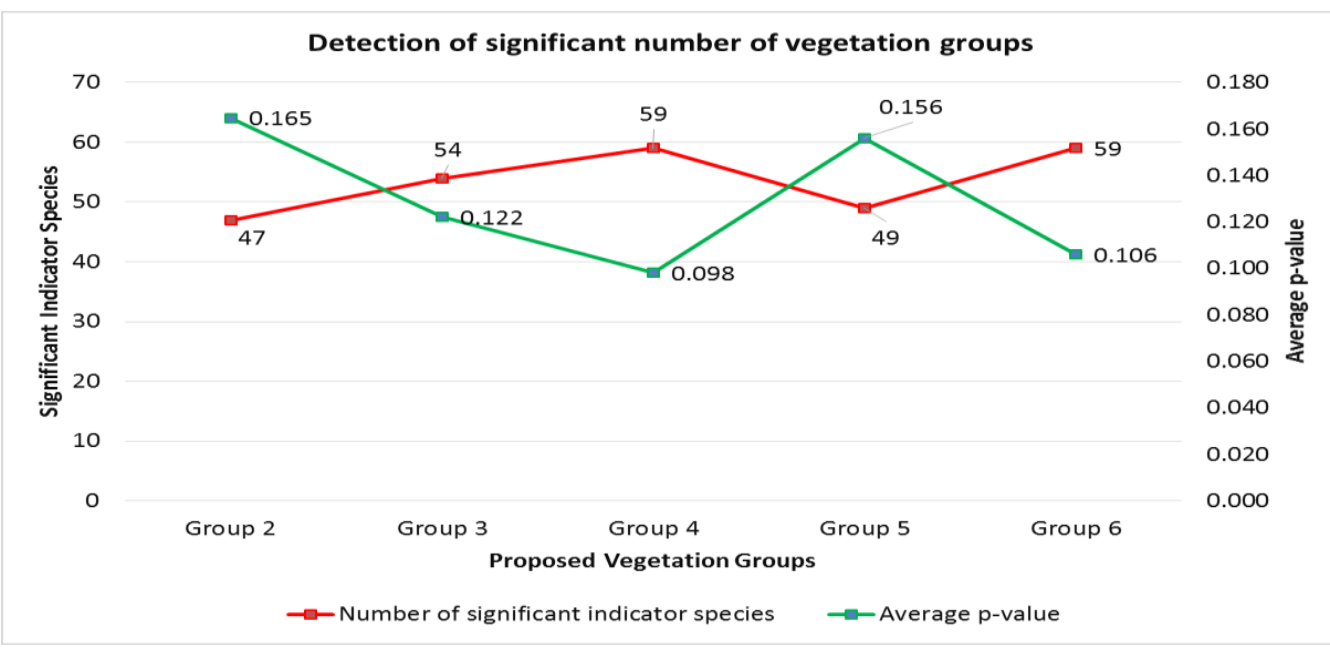

Figure 2. A graph showing the results of Monte Carlo test statistics used to decide the number of biologically significant vegetation groups in the study area 
Table 2. A table showing the results of pairwise comparisons (MRPP) of four (4) plant associations of the Sudhan Gali \& Ganga Hills

\begin{tabular}{ccc|c|c|c}
\hline \multicolumn{2}{c|}{ Plant communities compared } & T-statistic & A-value & p-value \\
\hline 1.BMD-18 & vs. & 2.PRP-6 & -13.63082095 & 0.20444995 & 0.00000015 \\
1.BMD-18 & vs. & 3.VAD-9 & -17.26453215 & 0.30257625 & 0.00000005 \\
1.BMD-18 & vs. & 4.STB-6 & -13.77931008 & 0.19771649 & 0.00000008 \\
2.PRP-6 & vs. & 3.VAD-9 & -9.15073886 & 0.37825685 & 0.00007066 \\
2.PRP-6 & vs. & 4.STB-6 & -6.65738026 & 0.30063123 & 0.00043338 \\
3.VAD-9 & vs. & 4.STB-6 & -7.99291567 & 0.26328556 & 0.00009218 \\
\hline \multicolumn{7}{c|}{ Overall } & & -21.590644 & 0.36049829 & 0.00000001 \\
\hline
\end{tabular}

For abbreviations see Table 4

\section{Vegetation types, composition and their key factors}

A total of three leading significant indicator species of each vegetation group were used to rename the vegetation groups as; 1 . Berberis-Micromeria-Desmodium (BMD) association, 2. Pinus-Rubus-Persicaria (PRP) association, 3. Viburnum-AbiesDryopteris (VAD) association and 4. Sibbaldia-Thymus-Bistorta (STB) association (Table 4). The details of each association is presented below:

\section{Berberis-Micromeria-Desmodium (BMD) community (sub-tropical chir-pine forest)}

This plant community was recorded at and around the Birpani town at an elevation range of 1700-2100 $\mathrm{m}$ (a), longitude (in dd) varied from 73.749-73.756 E, latitude from 34.046-34.066 N, and comprised of 18 samples/sub-stations (Fig. 3). This association was mostly located at the western faces (solar aspect) with moderate slope inclination $\left(22.49 \pm 5.73^{\circ}\right)$. A total of 63 plant species were recorded in this association out of which 41 appeared as indicator species (IS) and 20 as significant indicator species (SIS) of this community. The average species count per sample was $22.89 \pm 4.13$, and the absolute value varied from 14-30 in the studied samples. The leading ones were Berberis lycium, Micromeria biflora and Desmodium multiflorum. The least indicator value was recorded for Avena barbata and Solanum pseudocapsicum species. The Shannon's diversity index value was $2.87 \pm 0.18$, whereas Simpson's diversity index $(0.94 \pm 0.02)$, Inverse Simpson's/Fischer's diversity $(14.47 \pm 2.52)$, Pielou's evenness $(0.93 \pm 0.03)$, Margalef's richness $(5.05 \pm 0.68)$, and $\beta$-diversity (mean Euclidean distance: 67.3) were also recorded. The mean soil $\mathrm{pH}$ of this group was $7.22 \pm 0.61$, whereas soil EC $(\mathrm{mS} / \mathrm{cm})$, nitrate- $\mathrm{N}$ $(\mathrm{mg} / \mathrm{kg})$, phosphorus $(\mathrm{ppm})$, potassium $(\mathrm{ppm})$, and organic matter $(\%)$ values were $28 \pm 0.111,14 \pm 1.11,1.44 \pm 1.04,82.67 \pm 21.51$, and $2.25 \pm 0.61$ respectively. Some of the key factors of this association includes the highest values of mean soil $\mathrm{pH}$ (basic soils), species, richness, $\alpha$-diversities, and household density as compared to other associations. Similarly, the least values of altitude, nitrate-nitrogen, phosphorus, potassium, and organic matter, fuelwood collection (deforestation) and grazing pressure were other important factors (Tables 3 and 4). This scrubland association represents the remnants of stable Chir-pine forest, and extreme anthropogenic pressure in the past might be responsible of takeover of Berberis, Micromeria and Desmodium species. 


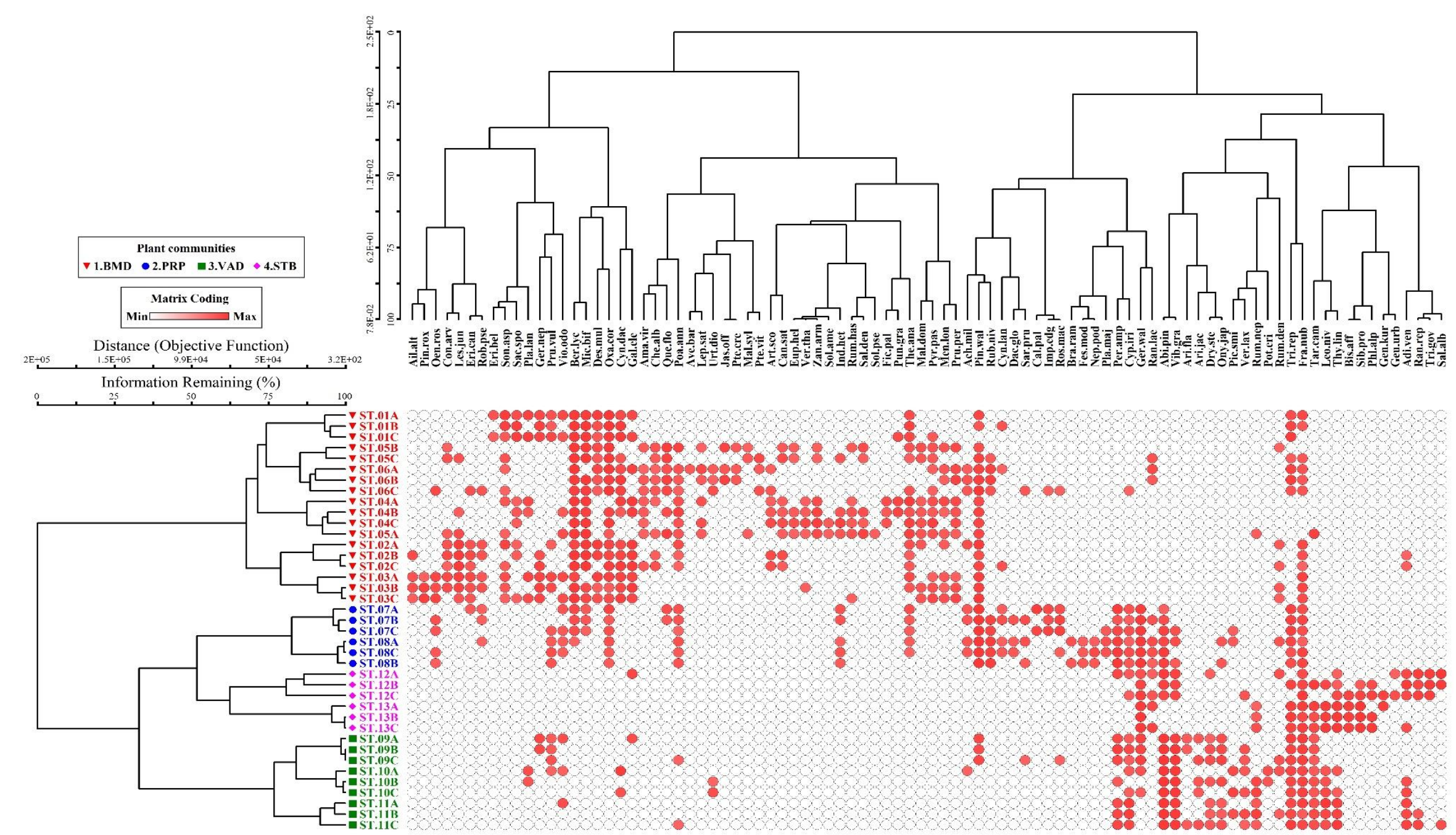

Figure 3. A clustering dendrogram showing the vegetation types, and their allied plant species of the western Himalayan forests of Sudhan Gali AJ\&K, Pakistan. (For species codes see Table 4) 
Table 3. A table showing the values (mean $\pm S D$ (min-max)) of studied factors of plant communities of the western Himalayan forests of Sudhan Gali, Azad Jammu and Kashmir, Pakistan

\begin{tabular}{|c|c|c|c|c|c|c|}
\hline & Variables & 1. BMD Association & 2. PRP Association & 3. VAD Association & 4. STB Association & All plots \\
\hline Loc & ality/ Samples/Plots & Birpani/18/162 & Sudhan Gali/6/54 & Upper Sudhan/9/81 & Ganga Top/6/54 & Study Area/39/351 \\
\hline \multicolumn{7}{|c|}{ Topographic variables } \\
\hline 1 & Altitude (m) & $\begin{array}{c}1834.95 \pm 100.72(1700- \\
2000)\end{array}$ & $\begin{array}{c}2151.67 \pm 53.72(2100- \\
2500)\end{array}$ & $\begin{array}{c}2601.34 \pm 86.48(2500- \\
2800)\end{array}$ & $\begin{aligned} 2950.67 & \pm 53.69(2900- \\
3000) & \end{aligned}$ & $\begin{array}{c}2232.17 \pm 437.47(1700- \\
3000)\end{array}$ \\
\hline 2 & Slope $\left(^{\circ}\right)$ & $\begin{array}{c}22.49 \pm 5.73(12.0491- \\
29.1633)\end{array}$ & $\begin{array}{c}34.13 \pm 6.49(28.2045- \\
40.0466)\end{array}$ & $\begin{array}{c}29.54 \pm 10.62(21.9847- \\
43.6736)\end{array}$ & $\begin{array}{c}20.6 \pm 8.23(13.088- \\
28.0975)\end{array}$ & $25.61 \pm 8.63(12.04-43.67)$ \\
\hline 3 & Slope aspect $\left(^{\circ}\right)$ & $\begin{array}{c}227.9 \pm 48.38(129.753- \\
271.975) \\
\end{array}$ & $\begin{array}{c}133.08 \pm 64.17(74.505- \\
191.646)\end{array}$ & $\begin{array}{c}197.54 \pm 139.28(12.588- \\
304.479)\end{array}$ & $\begin{array}{c}158.34 \pm 157.73(14.349- \\
302.32)\end{array}$ & $\begin{array}{c}195.59 \pm 100.06(12.58- \\
304.47)\end{array}$ \\
\hline 4 & Longitude (E) & $\begin{array}{c}73.753 \pm 0.003(73.749- \\
73.756) \\
\end{array}$ & $\begin{array}{c}73.744 \pm 0.002(73.742- \\
73.745) \\
\end{array}$ & $\begin{array}{c}73.759 \pm 0.006(73.753- \\
73.766) \\
\end{array}$ & $\begin{array}{c}73.787 \pm 0.001(73.787- \\
73.787) \\
\end{array}$ & $73.75 \pm 0.01(73.74-73.78)$ \\
\hline 5 & Latitude (N) & $\begin{array}{c}34.055 \pm 0.007(34.046- \\
34.066) \\
\end{array}$ & $\begin{array}{c}34.074 \pm 0.003(34.071- \\
34.076) \\
\end{array}$ & $\begin{array}{c}34.082 \pm 0.001(34.081- \\
34.083)\end{array}$ & $\begin{array}{c}34.077 \pm 0.001(34.076- \\
34.078) \\
\end{array}$ & $34.06 \pm 0.01(34.04-34.08)$ \\
\hline \multicolumn{7}{|c|}{ Diversity variables } \\
\hline 6 & Shannon's & $2.87 \pm 0.18(2.4-3.12)$ & $2.83 \pm 0.17(2.57-3.06)$ & $2.47 \pm 0.11(2.29-2.9)$ & $2.48 \pm 0.17(2.24-2.69)$ & $2.7 \pm 0.24(2.24-3.12)$ \\
\hline 7 & Simpson's & $0.94 \pm 0.02(0.9-0.95)$ & $0.93 \pm 0.02(0.9-0.94)$ & $0.89 \pm 0.02(0.85-0.93)$ & $0.91 \pm 0.02(0.89-0.93)$ & $0.91 \pm 0.02(0.85-0.95)$ \\
\hline 8 & Inverse Simpson's & $14.47 \pm 2.52(9.19-19.46)$ & $12.47 \pm 2.16(9.41-15.66)$ & $8.44 \pm 1.27(6.54-14.07)$ & $10.51 \pm 1.65(8.48-12.58)$ & $12.15 \pm 3.18(6.54-19.46)$ \\
\hline 9 & Pielou's & $0.93 \pm 0.03(0.88-0.97)$ & $0.9 \pm 0.02(0.86-0.91)$ & $0.88 \pm 0.03(0.83-0.91)$ & $0.95 \pm 0.04(0.88-0.97)$ & $0.91 \pm 0.03(0.83-0.97)$ \\
\hline 10 & Margalef's & $5.05 \pm 0.68(3.92-6.28)$ & $4.88 \pm 0.6(4.12-5.84)$ & $3.49 \pm 0.38(2.82-5.1)$ & $2.87 \pm 0.55(2.21-3.47)$ & $4.32 \pm 1.04(2.21-6.28)$ \\
\hline 11 & Average species & $22.89 \pm 4.13(14-30)$ & $23.50 \pm 3.08(20-29)$ & $17.11 \pm 1.62(14-19)$ & $14.17 \pm 2.56(11-17)$ & $20.3 \pm 4.77(11-30)$ \\
\hline 12 & Total species & 63 & 36 & 34 & 26 & 90 \\
\hline 13 & $\begin{array}{l}\text { Indicator species } \\
\text { (IS) }\end{array}$ & 41 & 21 & 14 & 14 & 90 \\
\hline 14 & $\begin{array}{c}\text { Significant indicator } \\
\text { species (SIS) }\end{array}$ & 20 & 17 & 10 & 12 & 59 \\
\hline
\end{tabular}




\begin{tabular}{|c|c|c|c|c|c|c|}
\hline \multicolumn{2}{|r|}{ Variables } & \multirow{2}{*}{$\frac{\text { 1. BMD Association }}{48.78}$} & \multirow{2}{*}{$\frac{\text { 2. PRP Association }}{80.95}$} & \multirow{2}{*}{$\begin{array}{c}\text { 3. VAD Association } \\
71.42 \\
\end{array}$} & \multirow{2}{*}{$\begin{array}{c}\text { 4. STB Association } \\
85.71 \\
\end{array}$} & \multirow{2}{*}{$\begin{array}{c}\text { All plots } \\
65.55\end{array}$} \\
\hline 15 & SIS to IS ratio & & & & & \\
\hline 16 & $\begin{array}{c}\beta \text {-diversity (mean } \\
\text { Euclidean distance) }\end{array}$ & 67.3 & 47.88 & 54.81 & 78.65 & 62.16 \\
\hline \multicolumn{7}{|c|}{ Edaphic variables } \\
\hline 17 & Soil pH. & $7.22 \pm 0.61(5.96-7.7)$ & $7.63 \pm 0.04(7.6-7.66)$ & $6.16 \pm 0.49(5.52-7.66)$ & $5.33 \pm 0.2(5.15-5.5)$ & $6.74 \pm 0.9(5.15-7.7)$ \\
\hline 18 & Soil EC & $0.28 \pm 0.11(0.17-0.45)$ & $0.24 \pm 0.04(0.21-0.27)$ & $0.29 \pm 0.1(0.17-0.39)$ & $0.28 \pm 0.24(0.07-0.49)$ & $0.27 \pm 0.11(0.07-0.49)$ \\
\hline 19 & Nitrate-N (mg/kg) & $1.14 \pm 1.11(0.43-3.5)$ & $0.84 \pm 0.08(0.77-0.9)$ & $4.76 \pm 1.68(2.75-6.62)$ & $4.3 \pm 2.8(1.75-6.85)$ & $2.41 \pm 2.25(0.43-6.85)$ \\
\hline 20 & Phosphorus (ppm) & $1.44 \pm 1.04(0.16-2.8)$ & $1.16 \pm 0.08(1.09-1.22)$ & $4.05 \pm 2.02(1.79-6.43)$ & $1.61 \pm 1.32(0.41-2.81)$ & $2.01 \pm 1.66(0.16-6.43)$ \\
\hline 21 & Potassium (ppm) & $82.67 \pm 21.51(56-118)$ & $108 \pm 52.59(60-156)$ & $183.34 \pm 86.75(80-280)$ & $67 \pm 3.29(64-70)$ & $107.38 \pm 62.96(56-280)$ \\
\hline 22 & Organic matter $(\%)$ & $2.25 \pm 0.61(1.1-2.9)$ & $2.2 \pm 0.22(2-2.4)$ & $3.47 \pm 1.39(2.4-5.3)$ & $2.65 \pm 0.06(2.6-2.7)$ & $2.58 \pm 0.9(1.1-5.3)$ \\
\hline \multicolumn{7}{|c|}{ Anthropogenic variables } \\
\hline 23 & $\begin{array}{l}\text { Grazing pressure } \\
\text { (dungs/ha) }\end{array}$ & $3.17 \pm 1.3(1-6)$ & $17.17 \pm 1.17(16-19)$ & $13.12 \pm 8.33(2-23)$ & $0.17 \pm 0.41(0-1)$ & $7.15 \pm 7.32(0-23)$ \\
\hline 24 & $\begin{array}{l}\text { Deforestation } \\
\text { pressure } \\
\text { (stumps/ha) }\end{array}$ & $5.23 \pm 2.19(1-9)$ & $4.34 \pm 2.07(2-6)$ & $14.78 \pm 2.54(11-19)$ & $0.5 \pm 0.84(0-2)$ & $6.56 \pm 5.18(0-19)$ \\
\hline 25 & $\begin{array}{l}\text { Forest types } \\
\text { (Champion et } \\
\text { al.,1965) }\end{array}$ & $\begin{array}{l}\text { Sub-Tropical Chir-Pine } \\
\text { Forest }\end{array}$ & $\begin{array}{c}\text { West Himalayan Moist } \\
\text { Temperate Blue-Pine } \\
\text { Forest }\end{array}$ & $\begin{array}{l}\text { West Himalayan Sub- } \\
\text { Alpine Fir Forest }\end{array}$ & $\begin{array}{l}\text { West Himalayan Alpine } \\
\text { Scrubland Forest }\end{array}$ & $\begin{array}{c}\text { West Himalayan Forest of } \\
\text { Sudhan Gali and Ganga } \\
\text { Summit }\end{array}$ \\
\hline 26 & Key factors & $\begin{array}{l}\text { Higher number of species, } \\
\text { richness, diversities, soil } \\
\text { pH (basic) \& human } \\
\text { population; moderate } \\
\text { grazing and deforestation } \\
\text { pressure; and least } \\
\text { elevation }\end{array}$ & $\begin{array}{l}\text { Higher average species } \\
\text { count in the plot, and } \\
\text { proportion of significant } \\
\text { indicators to indicators, } \\
\text { grazing pressure, slope, } \\
\text { soil pH (basic); mid- } \\
\text { elevation; and least } \\
\text { deforestation pressure }\end{array}$ & $\begin{array}{c}\text { Higher soil fertility (NPK), } \\
\text { organic matter, } \\
\text { deforestation, grazing, } \\
\text { proportion of significant } \\
\text { indicators to indicators } \\
\left(2^{\text {nd }}\right) \text {; and least richness, } \\
\text { diversities, and soil pH } \\
\text { (acidic) }\end{array}$ & $\begin{array}{c}\text { Highest elevation, } \beta \text { - } \\
\text { diversity, proportion of } \\
\text { significant indicators to } \\
\text { indicators; moderate NPK } \\
\text { \& organic matter; and least } \\
\text { slope, alpha-diversities, } \\
\text { richness, soil pH (acidic) }\end{array}$ & $\begin{array}{l}\text { The vegetation of the study } \\
\text { area is found under strong } \\
\text { influence of the local } \\
\text { environment }\end{array}$ \\
\hline
\end{tabular}


Table 4. A table showing the detailed results of indicator species analysis (ISA), vegetation types, species composition, and their significance

\begin{tabular}{|c|c|c|c|c|c|c|c|c|c|c|c|c|}
\hline \multirow{3}{*}{$\#$} & \multirow{3}{*}{ Latin Binomial/Common Name (English) } & \multirow{3}{*}{ Codes } & \multirow{3}{*}{ GF } & \multicolumn{4}{|c|}{ Groups (samples) } & \multirow{3}{*}{$\begin{array}{l}\text { Max. } \\
\text { Grp }\end{array}$} & \multirow{3}{*}{$\begin{array}{l}\text { Obs. } \\
\text { IV }\end{array}$} & \multirow{3}{*}{ Mean } & \multirow{3}{*}{ SD } & \multirow{3}{*}{ p-value } \\
\hline & & & & 1(18) & 2(6) & 3(9) & $4(6)$ & & & & & \\
\hline & & & & \multicolumn{4}{|c|}{ Indicator value (IV) } & & & & & \\
\hline 1 & Berberis lycium Royle/Indian Barberry & Ber.lyc & $\mathbf{N p}$ & 86 & 9 & $\mathbf{0}$ & $\mathbf{0}$ & 1 & 86 & 25.7 & 6.6 & 0.0002 \\
\hline 2 & $\begin{array}{l}\text { Micromeria biflora (Buch.-Ham. ex D.Don) Benth./ } \\
\text { Indian Wild Thyme }\end{array}$ & Mic.bif & Th & 81 & 4 & $\mathbf{0}$ & $\mathbf{0}$ & 1 & 81 & 24.4 & 7.2 & 0.0002 \\
\hline 3 & Desmodium multiflorum DC./Hawaiian Tick-trefoil & Des.mul & $\mathbf{N p}$ & 72 & $\mathbf{0}$ & $\mathbf{0}$ & $\mathbf{0}$ & 1 & 72 & 20.9 & 8 & 0.0004 \\
\hline 4 & Sonchus asper (L.) Hill/Spiny Sow Thistle & Son.asp & $\mathrm{H}$ & 72 & 0 & 0 & 0 & 1 & 72.2 & 21.5 & 8.4 & 0.0002 \\
\hline 5 & Oxalis corniculata $\mathrm{L} . /$ Creeping Woodsorrel & Oxa.cor & $\mathrm{H}$ & 67 & 29 & 0 & 0 & 1 & 67.2 & 26.9 & 6.94 & 0.0002 \\
\hline 6 & Pyrus pashia Buch.-Ham. ex D.Don/Indian Wild Pear & Pyr.pas & $\mathrm{Mp}$ & 67 & 0 & 0 & 0 & 1 & 66.7 & 20.2 & 8.12 & 0.0006 \\
\hline 7 & Cynodon dactylon (L.) Pers./Bermuda Grass & Cyn.dac & $\mathrm{H}$ & 66 & 0 & 5 & 0 & 1 & 65.9 & 22.9 & 7.2 & 0.0004 \\
\hline 8 & Themeda anathera (Nees ex Steud.) Hack./Kangaroo Grass & The.ana & $\mathrm{H}$ & 57 & 27 & 0 & 0 & 1 & 56.8 & 25.4 & 7.48 & 0.0016 \\
\hline 9 & Mentha longifolia (L.) L./Wild Horse Mint & Men.lon & $\mathrm{H}$ & 56 & 0 & 0 & 0 & 1 & 55.6 & 18.6 & 8 & 0.0018 \\
\hline 10 & Prunus persica (L.) Batsch./Common Peach & Pru.per & $\mathrm{Mp}$ & 56 & 0 & 0 & 0 & 1 & 55.6 & 18.5 & 7.9 & 0.0028 \\
\hline 11 & Saccharum spontaneum L./Wild Sugarcane & Sac.spo & G & 50 & 0 & 0 & 0 & 1 & 50 & 18.9 & 8.73 & 0.0082 \\
\hline 12 & $\begin{array}{c}\text { Lespedeza juncea } \text { (L.f.) Pers./ } \\
\text { Chinese Lespedeza, Silky Bush-Clover }\end{array}$ & Les.jun & $\mathrm{H}$ & 50 & 0 & 0 & 0 & 1 & 50 & 18.1 & 8.16 & 0.0038 \\
\hline 13 & Chenopodium album L./Lamb's Quarters, Fat Hen & Che.alb & Th & 50 & 0 & 0 & 0 & 1 & 50 & 17.8 & 8.02 & 0.0036 \\
\hline 14 & Amaranthus viridis L./Green Amaranth, Slender Amaranth & Ama.vir & Th & 50 & 0 & 0 & 0 & 1 & 50 & 17.6 & 7.93 & 0.0054 \\
\hline 15 & Malus domestica Borkh./Apple & Mal.dom & $\mathrm{Mp}$ & 50 & 0 & 0 & 0 & 1 & 50 & 17.6 & 7.83 & 0.0036 \\
\hline 16 & Galium elegans Wall. ex Roxb./Elegant Goose Grass & Gal.ele & Th & 48 & 0 & 1 & 2 & 1 & 48.3 & 20.5 & 7.65 & 0.008 \\
\hline 17 & Convolvulus arvensis L./Field Bindweed & Con.arv & $\mathrm{L}$ & 44 & 0 & 0 & 0 & 1 & 44.4 & 17.3 & 8.07 & 0.0118 \\
\hline 18 & Cannabis sativa L./Marijuana & Can.sat & Th & 44 & 0 & 0 & 0 & 1 & 44.4 & 17 & 7.92 & 0.011 \\
\hline 19 & Artemisia scoparia Waldst. \& Kitam./Red-stem Wormwood & Art.sco & $\mathrm{H}$ & 39 & 0 & 0 & 0 & 1 & 38.9 & 16.1 & 8.04 & 0.0182 \\
\hline 20 & Zanthoxylum armatum DC./Winged Prickly Ash & Zan.arm & $\mathrm{Np}$ & 33 & 0 & 0 & 0 & 1 & 33.3 & 15.7 & 8.25 & 0.0478 \\
\hline 21 & Lepidium sativum L./Common Garden Cress & Lep.sat & Th & 33 & 0 & 0 & 0 & 1 & 33.3 & 15.2 & 7.89 & 0.0452 \\
\hline 22 & Rumex hastatus D. Don/Arrow-Leaf Dock & Rum.has & $\mathrm{H}$ & 33 & 0 & 0 & 0 & 1 & 33.3 & 15.2 & 8.04 & 0.0502 \\
\hline
\end{tabular}




\begin{tabular}{|c|c|c|c|c|c|c|c|c|c|c|c|c|}
\hline \multirow{3}{*}{$\#$} & \multirow{3}{*}{ Latin Binomial/Common Name (English) } & \multirow{3}{*}{ Codes } & \multirow{3}{*}{ GF } & \multicolumn{4}{|c|}{ Groups (samples) } & \multirow{3}{*}{$\begin{array}{l}\text { Max. } \\
\text { Grp }\end{array}$} & \multirow{3}{*}{$\begin{array}{l}\text { Obs. } \\
\text { IV }\end{array}$} & \multirow{3}{*}{ Mean } & \multirow{3}{*}{ SD } & \multirow{3}{*}{ p-value } \\
\hline & & & & 1(18) & 2(6) & 3(9) & $4(6)$ & & & & & \\
\hline & & & & \multicolumn{4}{|c|}{ Indicator value (IV) } & & & & & \\
\hline 23 & Erigeron canadensis L./Canadian Horseweed & Eri.can & $\mathrm{Th}$ & 31 & 3 & 0 & 0 & 1 & 31.1 & 16.9 & 7.6 & 0.0528 \\
\hline 24 & Geranium nepalense Sweet/Nepalese Crane's Bill & Ger.nep & $\mathrm{H}$ & 30 & 0 & 7 & 0 & 1 & 30.3 & 18.1 & 7.86 & 0.075 \\
\hline 25 & Verbascum thapsus L./Great Mullein & Ver.tha & Th & 28 & 0 & 0 & 0 & 1 & 27.8 & 14.8 & 8.15 & 0.0692 \\
\hline 26 & Euphorbia helioscopia L./Sun Spurge & Eup.hel & Th & 28 & 0 & 0 & 0 & 1 & 27.8 & 14.5 & 8.01 & 0.0604 \\
\hline 27 & Salix denticulata Andersson/Elegant Willow & Sal.den & $\mathrm{Mp}$ & 28 & 0 & 0 & 0 & 1 & 27.8 & 14.5 & 8.07 & 0.0616 \\
\hline 28 & Quercus floribunda Lindl. ex A.Camus/Moru Oak, Green Oak & Que.flo & $\mathrm{Mp}$ & 27 & 10 & 0 & 0 & 1 & 27.2 & 17.6 & 7.82 & 0.118 \\
\hline 29 & Plantago lanceolata L./Ribwort Plantain & Pla.lan & $\mathrm{H}$ & 24 & 0 & 6 & 0 & 1 & 23.8 & 17 & 7.91 & 0.1814 \\
\hline 30 & Ailanthus altissima (Mill.) Swingle/Tree of Heaven & Ail.alt & $\mathrm{Mp}$ & 22 & 0 & 0 & 0 & 1 & 22.2 & 13.3 & 7.66 & 0.1772 \\
\hline 31 & Pinus roxburghii Sarg./Chir Pine, Roxburgh's Pine & Pin.rox & $\mathrm{Mp}$ & 17 & 0 & 0 & 0 & 1 & 16.7 & 13.2 & 6.8 & 0.2436 \\
\hline 32 & Malva sylvestris L./Common Mallow & Mal.syl & $\mathrm{Ch}$ & 17 & 0 & 0 & 0 & 1 & 16.7 & 12.5 & 7.2 & 0.2745 \\
\hline 33 & Jasminum officinale L./Common Jasmine & Jas.off & $\mathrm{Np}$ & 17 & 0 & 0 & 0 & 1 & 16.7 & 12.4 & 7.02 & 0.2509 \\
\hline 34 & Pteris vittata L./Chinese Laddar Brake & Pte.vit & G & 17 & 0 & 0 & 0 & 1 & 16.7 & 12.3 & 6.93 & 0.2577 \\
\hline 35 & Punica granatum L./Pomegranate & Pun.gra & $\mathrm{Mp}$ & 17 & 0 & 0 & 0 & 1 & 16.7 & 12.3 & 7.08 & 0.2851 \\
\hline 36 & Ficus palmata Forssk./Wild Fig & Fic.pal & $\mathrm{Mp}$ & 17 & 0 & 0 & 0 & 1 & 16.7 & 12.2 & 7.06 & 0.2629 \\
\hline 37 & Pteris cretica L./Cretan Brake & Pte.cre & G & 17 & 0 & 0 & 0 & 1 & 16.7 & 12.1 & 7.03 & 0.2851 \\
\hline 38 & Solanum americanum Mill./Black Nightshade & Sol.ame & Th & 11 & 0 & 0 & 0 & 1 & 11.1 & 11.8 & 5.42 & 0.5227 \\
\hline 39 & $\begin{array}{c}\text { Erigeron bellidioides (Buch.-Ham. ex D.Don) Benth. ex C.B.Clarke/Daisy } \\
\text { Fleabane }\end{array}$ & Eri.bel & $\mathrm{H}$ & 11 & 0 & 0 & 0 & 1 & 11.1 & 11.7 & 5.62 & 0.5065 \\
\hline 40 & Avena barbata Pott ex Link/Wild Oat & Ave.bar & Th & 6 & 0 & 0 & 0 & 1 & 5.6 & 10.1 & 4.77 & 1 \\
\hline 41 & Solanum pseudocapsicum L./Jerusalem Cherry & Sol.pse & $\mathrm{Th}$ & 6 & 0 & 0 & 0 & 1 & 5.6 & 10.1 & 4.77 & 1 \\
\hline 42 & $\begin{array}{l}\text { Pinus wallichiana A.B.Jacks./ } \\
\text { Blue Pine, Himalayan White Pine }\end{array}$ & Pin.wal & Mp & 22 & 72 & 2 & $\mathbf{0}$ & 2 & 72 & 28.3 & 6.4 & 0.0002 \\
\hline 43 & Rubus niveus Thunb./Ceylon Raspberry & Rub.niv & $\mathbf{N p}$ & 3 & 71 & $\mathbf{0}$ & $\mathbf{0}$ & 2 & 71 & 17.6 & 7.6 & 0.0002 \\
\hline 44 & $\begin{array}{l}\text { Persicaria amplexicaulis (D.Don) Ronse Decr./ } \\
\text { Mountain's Fleece }\end{array}$ & Per.amp & $\mathbf{H}$ & $\mathbf{0}$ & 64 & 23 & 1 & 2 & 64 & 21.6 & 8.1 & 0.0016 \\
\hline 45 & Rosa moschata Herrm./Musk Rose & Ros.mos & $\mathrm{Np}$ & 0 & 63 & 0 & 0 & 2 & 63.3 & 16.2 & 8.47 & 0.0012 \\
\hline
\end{tabular}




\begin{tabular}{|c|c|c|c|c|c|c|c|c|c|c|c|c|}
\hline \multirow{3}{*}{$\#$} & \multirow{3}{*}{ Latin Binomial/Common Name (English) } & \multirow{3}{*}{ Codes } & \multirow{3}{*}{ GF } & \multicolumn{4}{|c|}{ Groups (samples) } & \multirow{3}{*}{$\begin{array}{l}\text { Max. } \\
\text { Grp }\end{array}$} & \multirow{3}{*}{$\begin{array}{l}\text { Obs. } \\
\text { IV }\end{array}$} & \multirow{3}{*}{ Mean } & \multirow{3}{*}{ SD } & \multirow{3}{*}{ p-value } \\
\hline & & & & 1(18) & $2(6)$ & 3(9) & $4(6)$ & & & & & \\
\hline & & & & \multicolumn{4}{|c|}{ Indicator value (IV) } & & & & & \\
\hline 46 & Indigofera heterantha Brandis/Himalayan Indigo & Ind.het & $\mathrm{Np}$ & 6 & 60 & 0 & 0 & 2 & 60 & 17.8 & 8.04 & 0.0012 \\
\hline 47 & $\begin{array}{c}\text { Cynoglossum lanceolatum Forssk./ } \\
\text { Chinese Forget-Me-Not, Hound's Tongue }\end{array}$ & Cyn.lan & Th & 4 & 53 & 0 & 0 & 2 & 52.6 & 16.1 & 8.07 & 0.004 \\
\hline 48 & Geranium wallichianum D.Don ex Sweet/Buxton's Blue & Ger.wal & $\mathrm{H}$ & 0 & 52 & 6 & 37 & 2 & 51.8 & 23.1 & 7.47 & 0.0042 \\
\hline 49 & Nepeta podostachys Benth./Long Stalked Catmint & Nep.pod & $\mathrm{Ch}$ & 0 & 50 & 0 & 0 & 2 & 50 & 12.6 & 7.17 & 0.0048 \\
\hline 50 & Festuca modesta Steud./Fescue & Fes.mod & $\mathrm{H}$ & 0 & 50 & 0 & 0 & 2 & 50 & 12.4 & 7.13 & 0.0048 \\
\hline 51 & Dactylis glomerata L./Cock's Foot, Orchard Grass & Dac.glo & $\mathrm{H}$ & 0 & 50 & 0 & 0 & 2 & 50 & 12 & 6.9 & 0.0048 \\
\hline 52 & Plantago major L./Broad-Leaved Plantain, White-Man's Foot & Pla.maj & $\mathrm{H}$ & 0 & 50 & 0 & 0 & 2 & 50 & 12 & 6.93 & 0.0038 \\
\hline 53 & Impatiens edgeworthii Hook.f./Edgeworth's Balsam & Imp.edg & Th & 0 & 49 & 0 & 0 & 2 & 48.9 & 13.8 & 7.82 & 0.003 \\
\hline 54 & Achillea millefolium L./Common Yarrow & Ach.mil & $\mathrm{H}$ & 6 & 47 & 0 & 0 & 2 & 46.9 & 17.7 & 7.82 & 0.0064 \\
\hline 55 & Sarcococca pruniformis Lindl./Christmas Box, Sweet Box & Sar.pru & $\mathrm{Np}$ & 0 & 43 & 1 & 0 & 2 & 42.9 & 14.4 & 7.8 & 0.0066 \\
\hline 56 & Oenothera rosea L'Hér. ex Aiton/Evening Primrose & Oen.ros & $\mathrm{Ch}$ & 9 & 40 & 0 & 0 & 2 & 40.2 & 17 & 7.77 & 0.0178 \\
\hline 57 & Brachiaria ramosa (L.) Stapf./Brown-top Millet & Bra.ram & Th & 0 & 33 & 0 & 0 & 2 & 33.3 & 12 & 5.67 & 0.0452 \\
\hline 58 & Caltha palustris L./Yellow Marsh Marigold & Cal.pal & Th & 0 & 33 & 0 & 0 & 2 & 33.3 & 12 & 5.68 & 0.044 \\
\hline 59 & Prunella vulgaris L./Carpenter's Weed, Common Heal-All & Pru.vul & Th & 10 & 31 & 10 & 0 & 2 & 31.4 & 21.3 & 7.85 & 0.1086 \\
\hline 60 & Viola odorata L./Sweet Violet, English Violet & Vio.odo & $\mathrm{H}$ & 11 & 27 & 9 & 0 & 2 & 27.2 & 20.4 & 7.67 & 0.1718 \\
\hline 61 & $\begin{array}{c}\text { Picea smithiana (Wall.) Boiss./ } \\
\text { West Himalayan Spruce, Morinda Spruce }\end{array}$ & Pic.smi & Mp & 0 & 24 & 11 & 0 & 2 & 24.3 & 14.3 & 7.88 & 0.1028 \\
\hline 62 & Robinia pseudoacacia L./Black Locust & Rob.pse & $\mathrm{Mp}$ & 17 & 20 & 0 & 0 & 2 & 19.7 & 17 & 7.95 & 0.2523 \\
\hline 63 & Viburnum grandiflorum Wall. ex DC./Cranberry Bush & Vib.gra & Np & $\mathbf{0}$ & 4 & 75 & 8 & 3 & 75 & 22.3 & 7.8 & 0.0002 \\
\hline 64 & Abies pindrow (Royle ex D.Don) Royle/West Himalayan Fir & Abi.pin & Mp & $\mathbf{0}$ & 15 & 74 & 6 & 3 & 74 & 24.3 & 7.7 & 0.0002 \\
\hline 65 & Dryopteris stewartii Fraser-Jenk./Stewart's Wood Fern & Dry.ste & G & $\mathbf{0}$ & $\mathbf{0}$ & 68 & 2 & 3 & 68 & 17.2 & 8 & 0.0004 \\
\hline 66 & Onychium japonicum (Thunb.) Kunze/Carrot Fern & Ony.jap & G & 0 & 8 & 60 & 0 & 3 & 60.2 & 18 & 8.07 & 0.0016 \\
\hline 67 & Arisaema jacquemontii Blume/Cobra Lily & Ari.jac & G & 0 & 0 & 56 & 0 & 3 & 55.6 & 14 & 7.84 & 0.0008 \\
\hline 68 & Veronica laxa Benth./Lax Speedwell & Ver.lax & Th & 0 & 0 & 54 & 3 & 3 & 53.6 & 16.3 & 8.03 & 0.0026 \\
\hline 69 & Cyperus iria L./Rice Flat-Sedge & Cyp.iri & $\mathrm{G}$ & 0 & 34 & 50 & 3 & 3 & 50 & 24.4 & 8.4 & 0.0136 \\
\hline
\end{tabular}




\begin{tabular}{|c|c|c|c|c|c|c|c|c|c|c|c|c|}
\hline \multirow{3}{*}{$\#$} & \multirow{3}{*}{ Latin Binomial/Common Name (English) } & \multirow{3}{*}{ Codes } & \multirow{3}{*}{ GF } & \multicolumn{4}{|c|}{ Groups (samples) } & \multirow{3}{*}{ Max. } & \multirow{3}{*}{$\begin{array}{c}\text { Obs. } \\
\text { IV }\end{array}$} & \multirow{3}{*}{ Mean } & \multirow{3}{*}{ SD } & \multirow{3}{*}{ p-value } \\
\hline & & & & 1(18) & $2(6)$ & 3(9) & $4(6)$ & & & & & \\
\hline & & & & \multicolumn{4}{|c|}{ Indicator value (IV) } & & & & & \\
\hline 70 & Rumex nepalensis Spreng./Nepal Dock & Rum.nep & $\mathrm{Ch}$ & 0 & 0 & 40 & 13 & 3 & 39.8 & 18.4 & 8.59 & 0.0274 \\
\hline 71 & Taraxacum campylodes G.E.Haglund/Blow-Ball, Lion's Tooth & Tar.cam & $\mathrm{H}$ & 0 & 0 & 39 & 37 & 3 & 39.3 & 21.4 & 7.69 & 0.0298 \\
\hline 72 & Arisaema flavum (Forssk.) Schott/Yellow Cobra Lily & Ari.fla & $\mathrm{G}$ & 0 & 0 & 33 & 0 & 3 & 33.3 & 12.1 & 7.18 & 0.016 \\
\hline 73 & Trifolium repens L./White Clover & Tri.rep & $\mathrm{H}$ & 4 & 29 & 33 & 18 & 3 & 33 & 26.9 & 5.45 & 0.1438 \\
\hline 74 & Potentilla eriocarpa Wall. ex Lehm./Woolly-Fruit Cinquefoil & Pot.eri & Th & 0 & 0 & 22 & 0 & 3 & 22.2 & 11.9 & 5.45 & 0.0888 \\
\hline 75 & Urtica dioica L./Stinging Nettle & Urt.dio & $\mathrm{H}$ & 7 & 0 & 13 & 0 & 3 & 12.7 & 14.5 & 7.99 & 0.4693 \\
\hline 76 & Rumex dentatus L./Toothed Dock, Aegean Dock & Rum.den & $\mathrm{Ch}$ & 6 & 3 & 12 & 4 & 3 & 11.5 & 18.6 & 8.22 & 0.831 \\
\hline 77 & Sibbaldia procumbens L./Creeping Sibbaldia & Sib.pro & $\mathbf{H}$ & $\mathbf{0}$ & $\mathbf{0}$ & $\mathbf{0}$ & 83 & 4 & 83 & 14.5 & 7.9 & 0.0002 \\
\hline 78 & Thymus linearis Benth./Himalayan Thyme & Thy.lin & $\mathbf{H}$ & $\mathbf{0}$ & $\mathbf{0}$ & 18 & 67 & 4 & 67 & 19.8 & 8.4 & 0.0002 \\
\hline 79 & Bistorta affinis (D.Don) Greene/Himalayan Fleece Flower & Bis.aff & $\mathbf{H}$ & $\mathbf{0}$ & $\mathbf{0}$ & $\mathbf{0}$ & 67 & 4 & 67 & 12.9 & 7.4 & 0.0002 \\
\hline 80 & Phleum alpinum L./Alpine Cat's-Tail, Alpine Timothy & Phl.alp & G & 0 & 0 & 0 & 67 & 4 & 66.7 & 13.3 & 7.78 & 0.001 \\
\hline 81 & Poа апnиа L./Annual Meadow Grass, Annual Blue-Grass & Poa.ann & $\mathrm{H}$ & 5 & 9 & 22 & 58 & 4 & 58.4 & 30.8 & 5.82 & 0.0002 \\
\hline 82 & Trillium govanianum Wall. ex D.Don/Himalayan Trillium & Tri.gov & Th & 0 & 0 & 0 & 50 & 4 & 50 & 12.2 & 7.37 & 0.0062 \\
\hline 83 & Ranunculus repens L./Creeping Buttercup & Ran.rep & $\mathrm{H}$ & 0 & 0 & 5 & 40 & 4 & 39.7 & 14.2 & 8.03 & 0.0176 \\
\hline 84 & Leontopodium nivale subsp. alpinum (Cass.) Greuter/Edelweiss & Leo.niv & $\mathrm{H}$ & 0 & 0 & 28 & 39 & 4 & 39 & 18.9 & 8.25 & 0.0322 \\
\hline 85 & Adiantum venustum D. Don/Himalayan Maiden-Hair Fern & Adiven & $\mathrm{G}$ & 0 & 0 & 23 & 37 & 4 & 37.2 & 19.6 & 8.07 & 0.0402 \\
\hline 86 & $\begin{array}{l}\text { Fragaria nubicola (Lindl. ex Hook.f.) Lacaita/ } \\
\text { Wild Himalayan Strawberry }\end{array}$ & Fra.nub & $\mathrm{H}$ & 8 & 22 & 32 & 35 & 4 & 34.6 & 29.3 & 3.9 & 0.1016 \\
\hline 87 & $\begin{array}{c}\text { Ranunculus laetus Wall. ex Hook. f. \& J.W. Thomson/ } \\
\text { Cheerful Buttercup }\end{array}$ & Ran.lae & $\mathrm{H}$ & 2 & 26 & 0 & 34 & 4 & 34 & 19.5 & 8.12 & 0.0648 \\
\hline 88 & Gentiana kurroo Royle/Himalayan Gentian & Gen.kur & Th & 0 & 0 & 0 & 33 & 4 & 33.3 & 11.8 & 5.39 & 0.0392 \\
\hline 89 & Geum urbanum L./Herb Bennet, Wood Avens & Geu.urb & $\mathrm{H}$ & 0 & 0 & 0 & 33 & 4 & 33.3 & 11.8 & 6.14 & 0.0412 \\
\hline 90 & Salix alba L./White Willow & Sal.alb & $\mathrm{Mp}$ & 0 & 0 & 1 & 31 & 4 & 31.4 & 13 & 6.94 & 0.0456 \\
\hline
\end{tabular}

Three leading plant species of each plant community are in bold

GF: Growth Form; Mp: Megaphanerophyte; Np: Nanophanerophyte; Ch: Chamaephyte; Th: Therophyte; H: Hemicryptophyte; G: Geophyte; L: Lianas/Climbers;

Max. Grp: Maximum group; IV: Indicator value 


\section{Pinus-Rubus-Persicaria (PRP) community (Himalayan moist temperate forest)}

This community was studied at the Sudhan Gali at an elevation range of 2100$2500 \mathrm{~m}$ (a.), and comprised of 6 samples. It was located at 73.742-73.745 E longitude, and 34.071-34.076 N latitude (Fig. 3). This community was recorded at the south-eastern faces having maximum mean slope $(34.13 \pm 6.49$; range: 28.2 40.05) value. A total of 36 species with 21 IS and 17 SIS species were recorded in this group. The average species count was $23.50 \pm 3.08$ (20-29) for this association, and was highest as compared to all other associations but least $\beta$-diversity (mean Euclidean distance: 47.8) value depicting the homogeneity and least compositional dissimilarity amongst the studied samples (Table 3). It was dominated by Pinus wallichiana, Rubus niveus and Persicaria amplexicaulis, whereas the least IV was observed for Picea smithiana and Robinia pseudoacacia (Table 4). Total species (richness) and $\alpha$-diversities were lower than the BMD association but higher than the other two (VAD and STB) associations of the higher elevation areas. The mean values of edaphic variables including soil $\mathrm{pH}$, soil $\mathrm{EC}$, nitrate- $\mathrm{N}(\mathrm{mg} / \mathrm{kg})$, phosphorus $(\mathrm{ppm})$, potassium $(\mathrm{ppm})$, and organic matter $(\%)$ were $7.63 \pm 0.04$, $0.24 \pm 0.04,0.84 \pm 0.08,1.16 \pm 0.08,108 \pm 52.59$, and $2.2 \pm 0.22$, respectively. Some key factors of this association includes the highest values of slope, average species count in the plots, proportion of significant indicators to indicators $\left(2^{\mathrm{nd}}\right)$, basic soil, and grazing pressure $(17.17 \pm 1.17 \mathrm{dungs} / \mathrm{ha})$, and the least values of $\beta$ diversity, soil EC, nitrogen, phosphorus, and organic matter as compared to other associations (Tables 3 and 4).

\section{Viburnum-Abies-Dryopteris (VAD) community (Himalayan sub-alpine forest)}

This association was studied at upper Sudhan Gali areas and an elevation range of 2500-2800 m (a.), comprised of 9 samples (81 plots). It was located at 73.753 73.766 E longitude, and 34.081-34.083 N latitude (Fig. 3). It was studied at north western faces with mean slope value of $29.54 \pm 10.62^{\circ}$ (range: $21.98-43.67^{\circ}$ ). It was comprised of 34 species, 14 indicator species and 10 significant indicator species. The leading significant ones were Viburnum grandiflorum, Abies pindrow, and Dryopteris stewartii. The species with minimum IV were Urtica dioica and Rumex dentatus (Tables 3 and 4). Total species (richness) and $\alpha$-diversities were further decreased as compared to both BMD and PRP associations of low elevation areas, and were almost alike to STB association of the higher elevation areas of the Ganga summit. The mean values of edaphic variables including soil $\mathrm{pH}$, soil $\mathrm{EC}$, nitrate-N $(\mathrm{mg} / \mathrm{kg})$, phosphorus $(\mathrm{ppm})$, potassium $(\mathrm{ppm})$, and organic matter $(\%)$ were $6.16 \pm 0.49,0.29 \pm 0.1,4.76 \pm 1.68,4.05 \pm 2.02,183.34 \pm 86.75$, and $3.47 \pm 1.39$ respectively. The grazing (dungs/ha) and deforestation (stumps/ha) pressure values were $13.12 \pm 8.33$ (2-23), and $14.78 \pm 2.54$ (11-19) respectively. Key factors of this association includes the highest values of soil EC, soil fertility (NPK), organic matter, vegetation cover, deforestation and higher grazing pressure (2nd), and proportion of significant indicators to indicators species. Similarly, least values of indicator species, SIS, $\alpha$-diversities, evenness, and relatively lower soil $\mathrm{pH}$ (acidic) values were other important factors (Tables 3 and 4). The interactions of the least values of soil $\mathrm{pH}$, higher soil fertility in term of NPK and thickest vegetation cover variables are all very important and correlated. The core areas of this association 
were least diverse with conifer dominancy, and no human settlements, therefore recorded under severe deforestation pressure and activities of the timber mafia.

\section{Sibbaldia-Thymus-Bistorta (STB) community (Himalayan alpine scrubland forest)}

This plant association was recorded at and around Ganga summit areas, elevation ranged from 2900 to $3000 \mathrm{~m}$ (a.), and comprised of 6 samples. It was located at 73.787 E longitude, and 34.076-34.078 N latitude (Fig. 3). This vegetation cluster was comprised of a total of 26 species, 14 indicator species, and 12 significant indicator species (Tables 3 and 4). The proportion of significant indicators to indicators was highest in this community, therefore, representing the uniqueness of this microhabitat in the area, and the important determining factors includes high altitude and associated least mean minimum temperatures. Three leading significant indicator species of this community were Sibbaldia procumbens, Thymus linearis, and Bistorta affinis, whereas the least IV was recorded for Gentiana kurroo, Geum urbanum and Salix alba species. During the ecological surveys, it was also observed that local inhabitants visit the areas of this association frequently during the growing season for collection of variety of medicinal plant species. Similarly, except Margalef's richness $(2.87 \pm 0.55)$, all other diversity values (viz. Shannon's diversity $2.48 \pm 0.17$; Simpson's diversity $0.91 \pm 0.02$; Simpson's reciprocal diversity $10.51 \pm 1.65$; Pielou's evenness $0.95 \pm 0.04$; and $\beta$-diversity 78.65 ) were higher than the previous sub-alpine VAD vegetation type, and lower than the first two (viz. BMD and PRP) vegetation types of low elevation area (Tables 3 and 4). This might be due to increase of anthropogenic activities (other than deforestation) above the timberline in the area. The mean values of edaphic variables including soil $\mathrm{pH}$, soil EC, nitrate- $\mathrm{N}(\mathrm{mg} / \mathrm{kg})$, phosphorus $(\mathrm{ppm})$, potassium $(\mathrm{ppm})$, and organic matter $(\%)$ were $5.33 \pm 0.2,0.28 \pm 0.24,4.3 \pm 2.8,1.61 \pm 1.32,67 \pm 3.29$, and $2.65 \pm 0.06$ respectively.

The key factors controlling this association were the highest values of altitude, evenness, $\beta$-diversity, and proportion of significant indicator to indicator species, and the least values of slope, total species (richness), average species, IS, SIS, soil $\mathrm{pH}$, and potassium contents of the soil (Tables 3 and 4).

\section{Ordination analyses}

\section{Detrended correspondence analysis}

The gradient length in the compositional response data was above 4 SD (standard deviations of species turnover) units, therefore, unimodal ordination models like DCA and CCA were analyzed. DCA was used to confirm the results of hierarchical clustering of the vegetation samples collected from the western Himalayan forests of Sudhan Gali, Pakistan. Total variations in the species response data were 2.84, whereas the first four axes cumulatively represents $37.3 \%$ variations. The gradient length of the first four DCA-axes was 4.4, 2.09, 2.49, and 1.25 respectively. DCA biplot of the plant species and studied samples allied to four vegetation types showed that both species and samples are reasonably separated in the ordination space (Fig. 4). Along the DCA axis-1, the plant species of the low elevation areas are distributed in the left half and species of higher elevation areas in the right hand side of the biplot. This species distribution pattern depicted the prominent role of altitude in the area. Secondly, this biplot is also depicting the pattern of diversity 
variations along the DCA axis-1 (or altitude), which continuously decreased up to VAD association and then again increased for the alpine STB association (Fig. 4).

\section{Canonical correspondence analysis (CCA) and variation partitioning}

The contribution of five topographic (altitude, Cos-trans aspect, slope, latitude, and longitude), six edaphic (soil $\mathrm{pH}$, organic matter, $\mathrm{EC}, \mathrm{N}, \mathrm{P}$, and $\mathrm{K}$ ), and two anthropogenic (grazing pressure, and deforestation) variables toward explaining the variations in the response data were tested by using CCA. All the richness, evenness, and diversity variables were not included in this test. The CCA model (overall) was detected as significant (Pseudo F-value $=4.61 ; \mathrm{p}$-value $=0.001$ ). Therefore, all the 13 constrained axes and variables were further tested by using permutation test. The results showed that first seven (7) CCA axes were significant (p-value $\leq 0.003$ ) (Fig. 5). Out of total inertia of 2.84 of the response data, all the explanatory variables explained $73.1 \%$ variations, whereas the adjusted explained variations were 59.2\%. First four CCA axes cumulatively explained $50.15 \%$ and $68.57 \%$ of variations and fitted variations respectively. A very high values of species-environment pseudo-canonical correlations of the first four CCA axes were observed (viz. r 0.99, 0.98, 0.97 and 0.92). This showed that the selected explanatory variables were determinants of the response (species) data variations. Similarly, the high values of species-environment correlation for the CCA axes 2-4 indicated that there was no single dominant environmental gradient in this part of the western Himalaya. By using permutation test, both simple (marginal) and conditional (net) term effects were tested for all the 13 explanatory variables. The results of simple term effect testing revealed that only the soil EC was not much important towards explaining variations in the species data, whereas the net effect testing made the same as significant as well (Table 5). The ranking (order of importance) results of the studied variables depicted that altitude is the leading factor followed by grazing pressure, deforestation, nitrate- $\mathrm{N}$, soil $\mathrm{pH}$, potassium, slope, longitude, phosphorus, cos-trans aspect, organic matter, latitude, and soil EC in the area (Table 5).

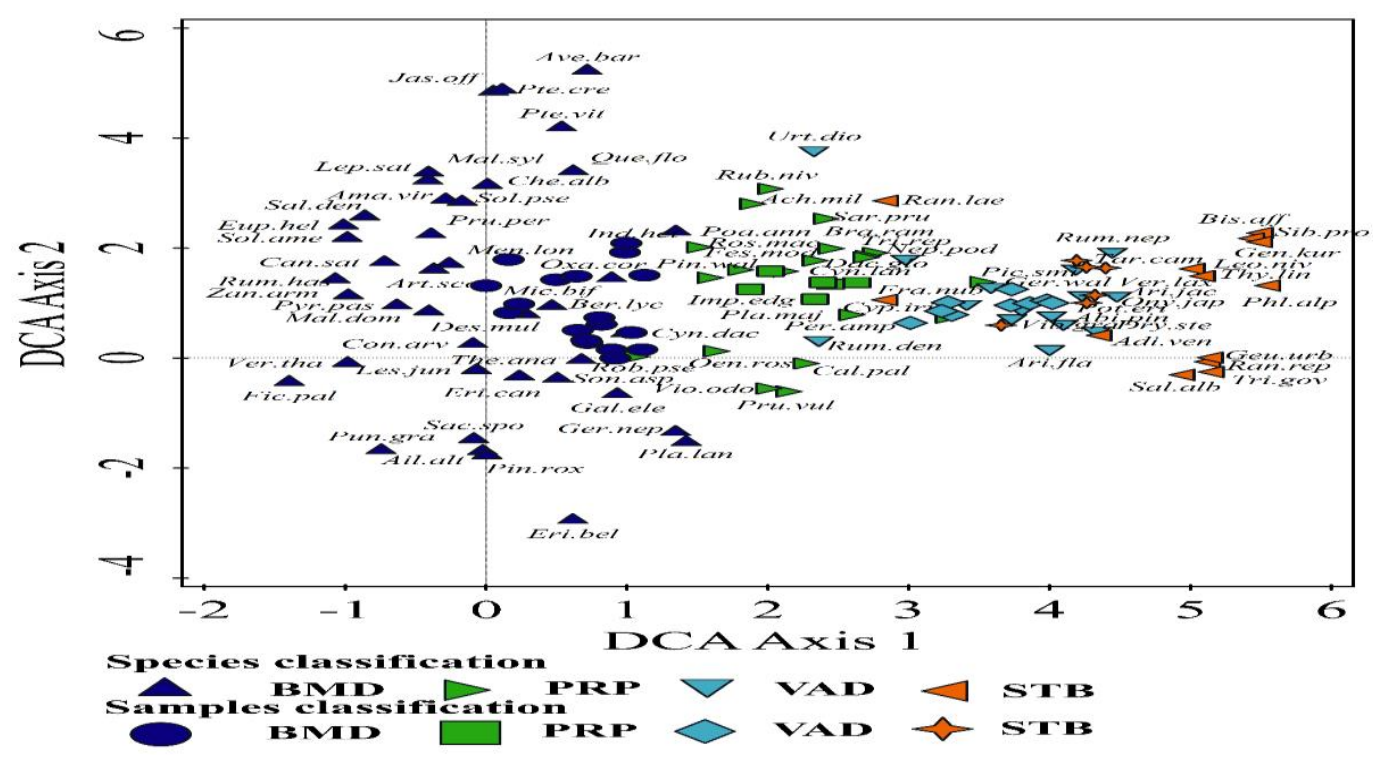

Figure 4. A DCA biplot showing distribution pattern of 39 samples and 90 plant species in four vegetation groups of western Himalayan forests of Sudhan Gali 


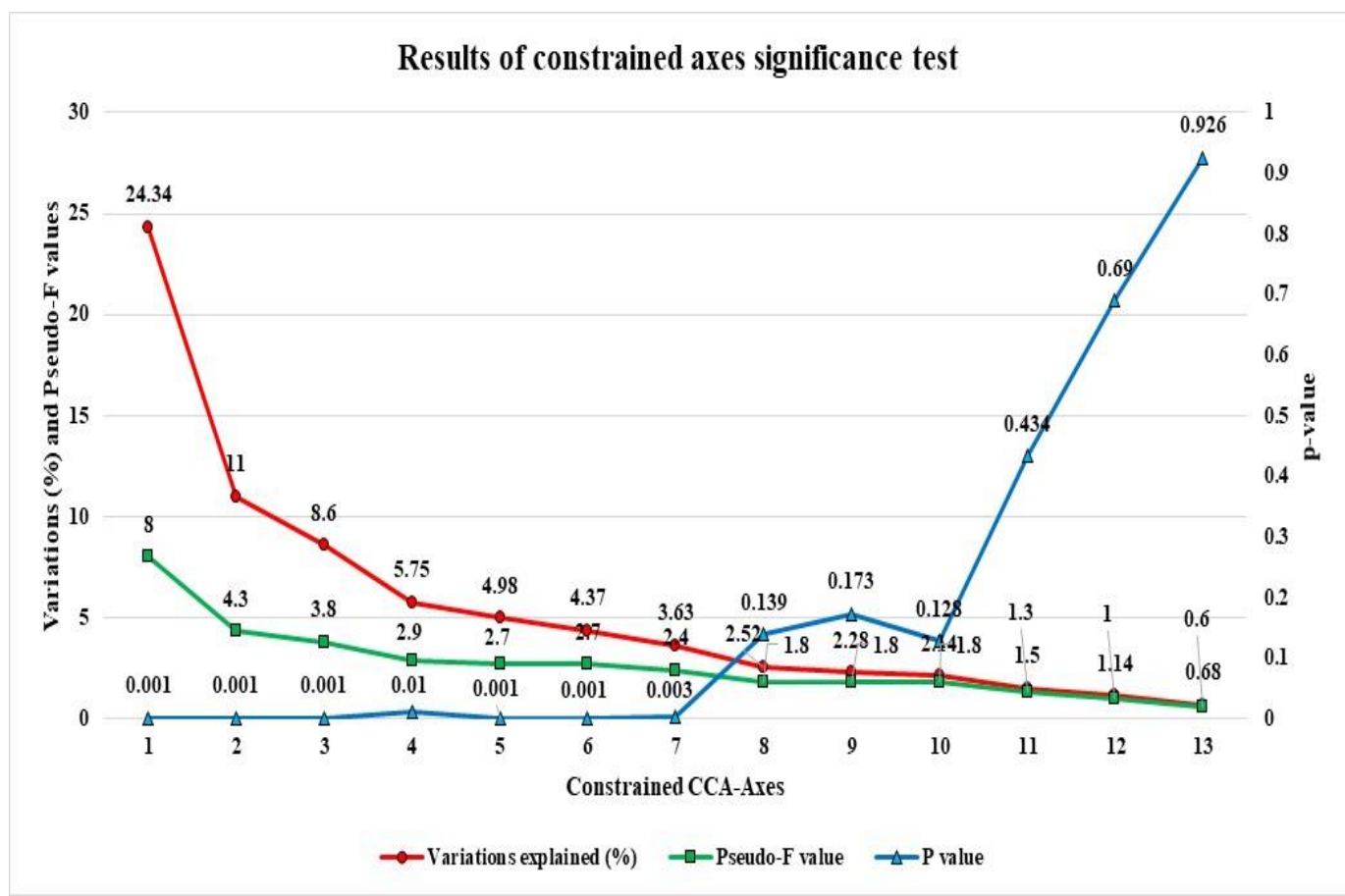

Figure 5. A plot showing the results of significance testing of 13 constrained axes

Table 5. A table showing the contribution and ranking of the studied environmental variables ( $p$-values were corrected by false discovery rate method)

\begin{tabular}{c|c|c|c|c|c|c|c|c|c}
\hline \multicolumn{4}{c}{ Simple term effects: } & \multicolumn{5}{c}{ Conditional term effects: } \\
\hline Variable & Explains \% & Pseudo-F & P-value & P(adj) & Variable & Explains \% & Pseudo-F & P-value & P(adj) \\
\hline Altitude & 23.2 & 11.2 & 0.001 & 0.0028 & Altitude & 23.2 & 11.2 & 0.001 & 0.0014 \\
Nitrate-N & 15.3 & 6.7 & 0.001 & 0.0028 & Grazing pressure & 9 & 4.8 & 0.001 & 0.0014 \\
Soil pH & 12.6 & 5.4 & 0.001 & 0.0028 & Deforestation & 6.4 & 3.7 & 0.001 & 0.0014 \\
Cos-trans Aspect & 9.4 & 3.9 & 0.001 & 0.0028 & Nitrate-N & 4 & 2.4 & 0.002 & 0.00233 \\
Grazing pressure & 9.1 & 3.7 & 0.001 & 0.0028 & Soil pH & 3.9 & 2.4 & 0.003 & 0.003 \\
Deforestation & 8.5 & 3.4 & 0.004 & 0.007 & Potassium & 3.5 & 2.2 & 0.003 & 0.003 \\
Potassium & 7.2 & 2.9 & 0.002 & 0.00467 & Slope & 4.7 & 3.3 & 0.001 & 0.0014 \\
Phosphorus & 6.9 & 2.7 & 0.004 & 0.007 & Longitude & 4.2 & 3.1 & 0.001 & 0.0014 \\
Organic matter & 6 & 2.4 & 0.012 & 0.01527 & Phosphorus & 3.8 & 2.9 & 0.001 & 0.0014 \\
Latitude & 6 & 2.3 & 0.008 & 0.01244 & Cos-trans Aspect & 3 & 2.5 & 0.001 & 0.0014 \\
Slope & 5.9 & 2.3 & 0.009 & 0.0126 & Organic matter & 2.9 & 2.5 & 0.001 & 0.0014 \\
Longitude & 5.9 & 2.3 & 0.014 & 0.01633 & Latitude & 2.7 & 2.4 & 0.002 & 0.00233 \\
Soil EC & 4 & 1.5 & 0.092 & 0.092 & Soil EC & 2.7 & 2.9 & 0.001 & 0.0014 \\
\hline
\end{tabular}

The graphical results of the CCA (first three CCA axes) are presented in Figure 6. These CCA biplots revealed that the species distribution of the four detected associations are controlled by the studied environment. The species of STB community got the maximum score along CCA axis 1, and the species of BMD community got the least, therefore primarily controlled by altitude, followed by $\mathrm{N}, \mathrm{P}, \mathrm{K}$, grazing and organic matter. Similarly, BMD and PRP associations are found separated along CCA axis-2, and controlled by deforestation, soil pH, longitude and slope variables (Fig. 6). 


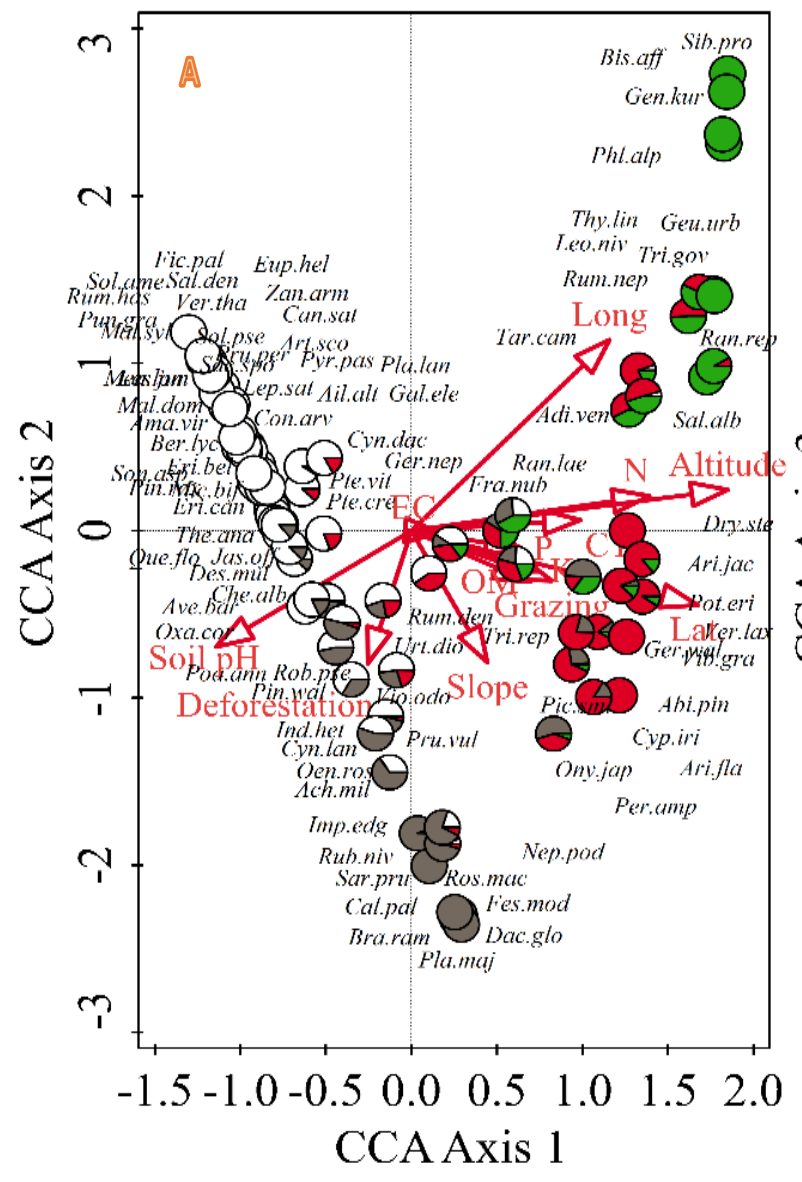

Environmental Variables $\gg$ Vegetation Asss $\square$ BMD $\square$ PRP $\square$ VAD $\square$ STB

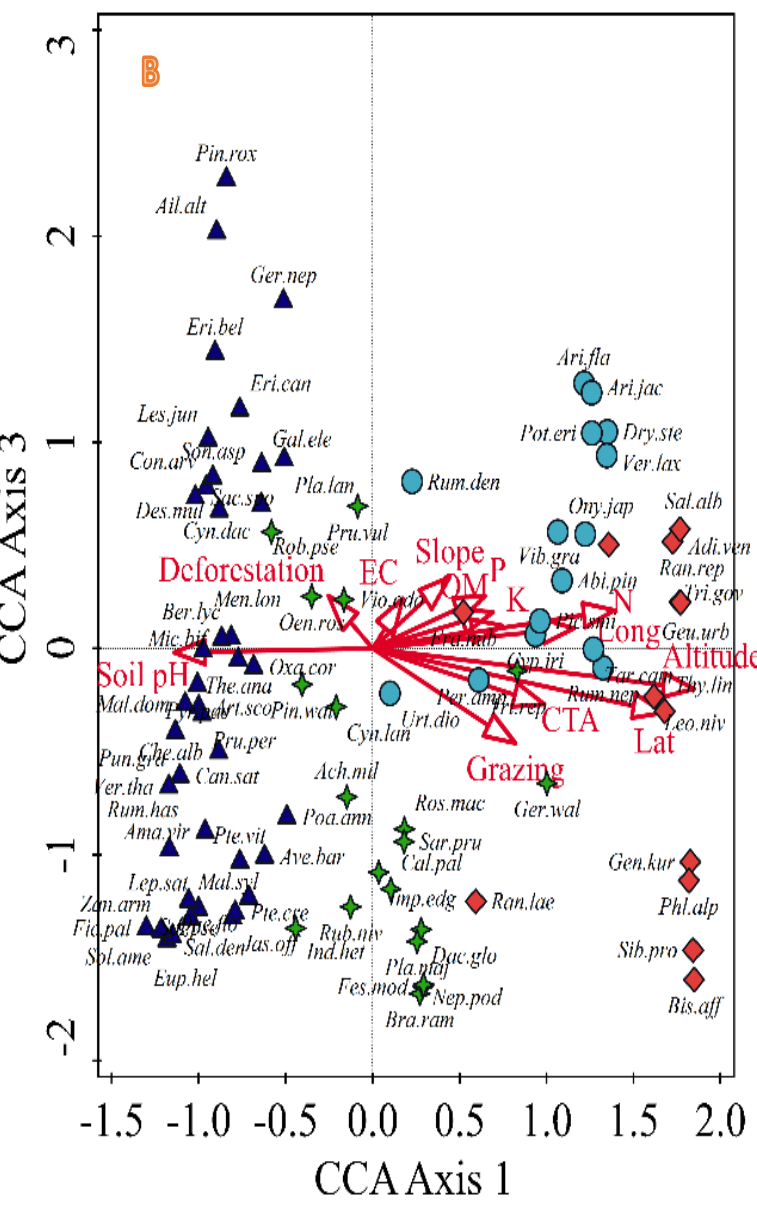

Environmental Variables $\gg$ Species classification $\triangle$ BMD $\diamond$ PRP $\bigcirc$ VAD $\diamond$ STB

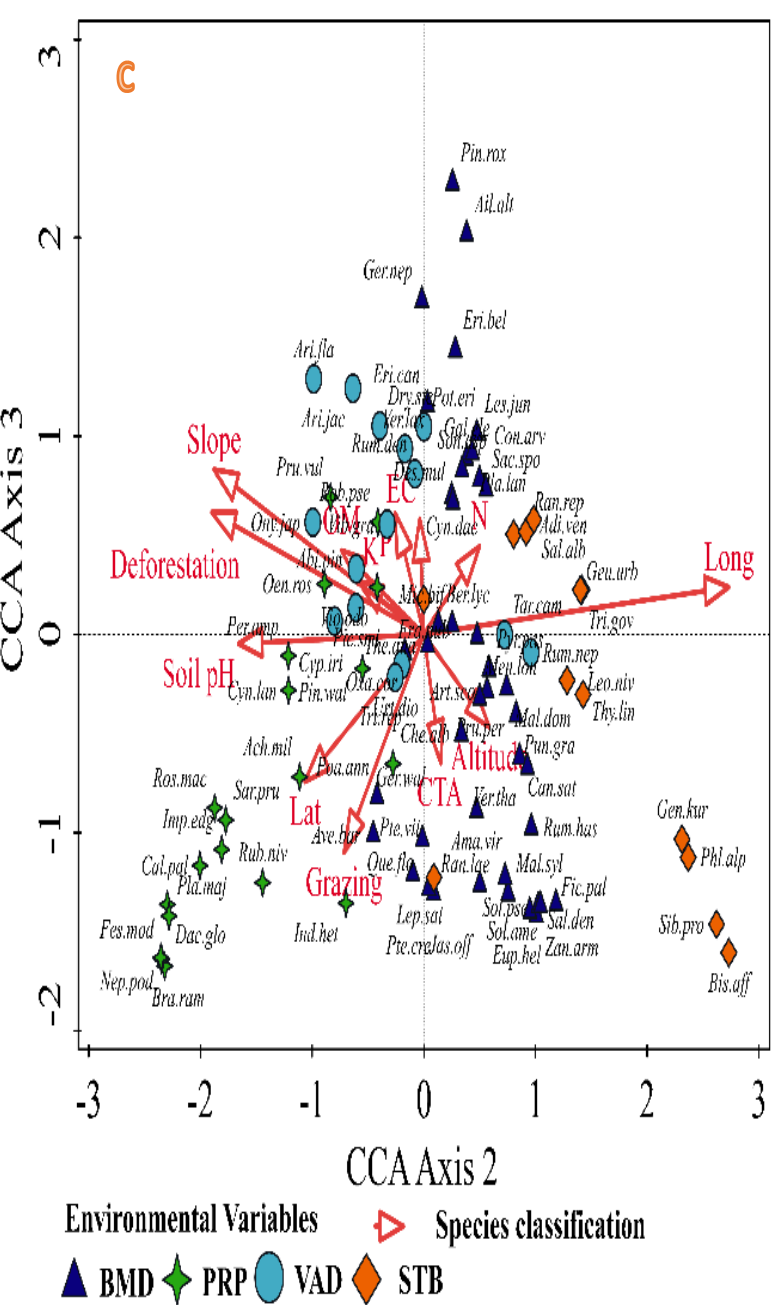

Environmental Variables $\triangleright$ Species classification

$\Delta$ BMD $\diamond \mathrm{PRP} \bigcirc \mathrm{VAD} \diamond \mathrm{STB}$

Figure 6. A group of CCA biplots depicting the species-environment relationships of the four vegetation types of the western Himalayan forests of Sudhan Gali, AJ\&K, Pakistan (CCA axes: $A=1 \& 2 ; B=1 \& 3 ; C=2 \& 3$ ) 
A total of two leading factors of each of topographic (altitude and slope), edaphic (nitrate- $\mathrm{N}$ and soil $\mathrm{pH}$ ), and anthropogenic (grazing pressure and deforestation) class were used to perform variation partitioning (partial CCA) to seek the contribution of each class and their interactions. Like CCA, permutation test was performed for net effects of each class. This analysis results depicted that topography was the leading (explained variations 32.6\%) class, followed by anthropogenic (25.8\%), and topographic vs. edaphic interaction $(25.5 \%)$. Similarly, least variations were explained by the interactions of all classes $(-5.5 \%)$. This negative value of the adjusted explained variations can be interpreted as zero, and linked to the samples where the explanatory variables explain less variations than what the random normal variables do (Fig. 7). However, at this moment, higher contribution of anthropogenic than the edaphic class is a major concern in the study area.

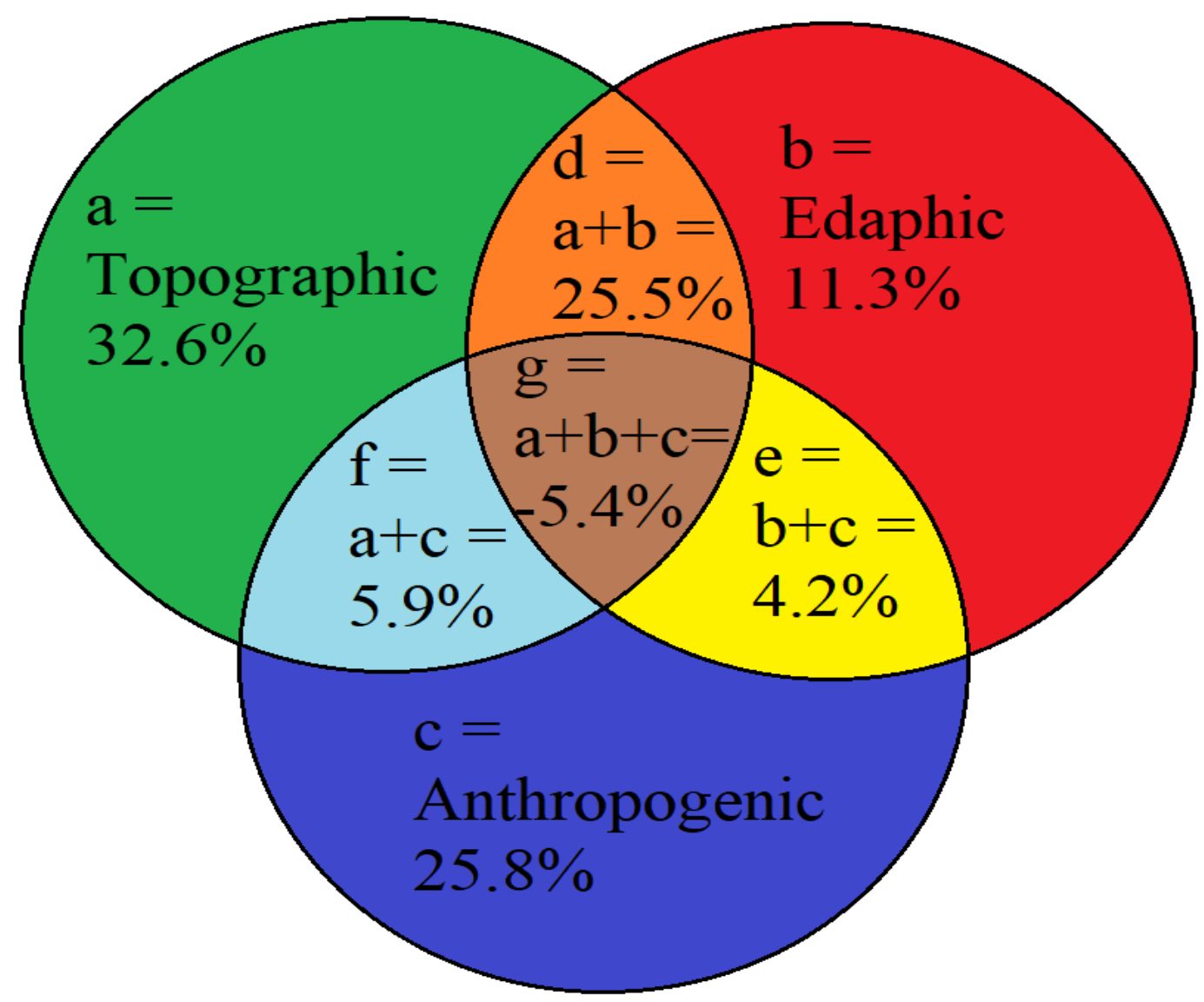

Figure 7. A Venn diagram showing the amount of explained variations (\%) in the species data by the three groups of explanatory variables

\section{Discussion}

A detailed ecological exploration of the western Himalayan forests is necessary for future biodiversity management, sustainable utilization and conservation (Shaheen et al., 2012; Khan et al., 2019). This study thoroughly and comparatively reviewed the history of forest ecological work conducted at the Sudhan Gali and Ganga summit of Bagh, AJ\&K, Pakistan, and identified few research gaps that needed to be explored. Prior to this study, a total of 14 different ecological studies were conducted from 2007 to 2018, but a lot 
of questions remained un-answered (Table 6). Therefore, this study represents the first ever detailed ecological exploration of the area. Majority of earlier studies rarely used agglomerative hierarchical clustering for classification, and mostly used a linear unconstrained model (PCA) for arranging the species along the environmental gradients. The drawback of using PCA include the Horseshoe Effect (an artifact of the ordination diagram similar to the Arch Effect), therefore, PCA axis-2 did not truly represent the secondary gradient. A study close to this one was conducted by Shaheen et al. (2012) in the study area, however, Monte Carlo permutation test to decide the ecological meaningful number of vegetation types, significant indicator species of each type by using indicator species analysis, pairwise compositional significance of the plant associations, contribution of edaphic variables, order of importance of studied environment, and variation partitioning were performed for the first time from the study area.

A total of four plant associations (1. Berberis-Micromeria-Desmodium (BMD), 2. Pinus-Rubus-Persicaria (PRP), 3. Viburnum-Abies-Dryopteris (VAD) and 4. SibbaldiaThymus-Bistorta (STB) associations) were recorded in this study, and confirmed by using different multivariate statistics. Sher et al. (2013) studied the vegetation at 13 different stations along the altitudinal gradient from the Sudhan Gali and Ganga area like this study, but communicated the composition of each station as a plant community and further place them in the higher hierarchy of four plant associations. Secondly, the elevation gradient of their studied stations was ranged from 2200 to $2500 \mathrm{~m}$ (a.) unlike this study which ranges from 1700 to $3000 \mathrm{~m}$ (a.). Malik and Malik (2012) also recorded four plant associations from the same area. Both these earlier studies did not employ any multivariate analysis tool. Therefore, a sufficient compositional differences were observed while comparing this with both prior studies. Unlike this study, both prior studies also communicated that there is no significant correlation between species turnover and altitudinal variations. However, the status of overall dominant species (especially Pinus wallichiana and Abies pindrow) in the area are same. The composition of alpine meadow (Ganga) matches with the results of Shaheen et al. (2011c), who also reported the similar community composition and leading dominants in the other areas of district Bagh. We recorded low indicator values of Pinus roxburghii and Pinus wallichiana (Blue Pine) at the lower elevation in the BMD vegetation type. Khan et al. (2019) reported the dominancy of Chir pine at the low elevation areas of adjacent Muzaffarabad district, whereas this study detected the dominancy of Berberis lycium and Micromeria biflora at the similar elevation areas. This might be due to higher rate of fuelwood collection and consumption than regeneration as compared to other related adjacent areas (Shaheen et al., 2011b). The other contributing factor might include the findings of Saeeda and Zakir (2012), which communicated the highest correlation between forest deterioration and poor socioeconomic status of the inhabitants of the Bagh area. The mid-elevation areas (2100-2500 m) of PRP vegetation type is relatively more stable than the other three types, but higher grazing pressure needs immediate attention. The shrub species of Viburnum grandiflorum has taken over the Abies pindrow and Picea smithiana in the sub-alpine VAD type (elevation: $2500-2800 \mathrm{~m}$ ). This type was recorded under sever deforestation pressure, possibly because located far from the human settlements, therefore a relatively easy target for timber mafia. Secondly, the local government has completed a new metallic road up to $2700 \mathrm{~m}$ elevation, and this provide an easy transportation tool for illegally obtained forest resources. The study area (especially STB type: 2900-3000 m is receiving a huge number of tourist's visits especially during the growing season which sometime get involved in uprooting of rare medicinal species. 


$$
-15499 \text { - }
$$

Table 6. A table showing the comparative history of vegetation explorations of the western Himalayan forests of Sudhan Gali \& Ganga summit, AJ\&K, Pakistan

\begin{tabular}{|c|c|c|c|c|c|c|c|c|c|}
\hline \multirow[t]{2}{*}{ No } & \multirow[t]{2}{*}{ Reference } & \multirow[t]{2}{*}{ Major research objective } & \multicolumn{3}{|c|}{$\begin{array}{l}\text { Environment } \\
\text { studied }\end{array}$} & \multirow[t]{2}{*}{$\mathbf{H C}$} & \multirow[t]{2}{*}{ ISA } & \multirow[t]{2}{*}{ ORD } & \multirow[t]{2}{*}{ Remarks/research gap } \\
\hline & & & $\mathbf{T}$ & $\mathbf{S}$ & D & & & & \\
\hline 1 & $\begin{array}{l}\text { Malik et al., } \\
2007\end{array}$ & \begin{tabular}{|c|} 
Raunkiaer's life forms and \\
leaf spectra classification
\end{tabular} & $x$ & $\times$ & $\times$ & $x$ & $x$ & $\times$ & Environmental influence is missing. \\
\hline 2 & $\begin{array}{c}\text { Siddiqui et al., } \\
\text { 2010a }\end{array}$ & Vegetation classification & $\checkmark$ & $\times$ & $\times$ & $\times$ & $x$ & PCA & $\begin{array}{l}\text { Environmental influence is missing. Cedrus } \\
\text { deodara is recorded as dominant, presently it } \\
\text { is not recorded in any sample }\end{array}$ \\
\hline 3 & \begin{tabular}{|c|} 
Siddiqui et al., \\
$2010 \mathrm{~b}$
\end{tabular} & $\begin{array}{l}\text { Vegetation-environment } \\
\text { interactions }\end{array}$ & $\checkmark$ & $\checkmark$ & $\times$ & $\checkmark$ & $\times$ & DCA & $\begin{array}{l}\text { Recorded environmental variables were not } \\
\text { included in DCA as supplementary variables }\end{array}$ \\
\hline 4 & \begin{tabular}{|c|} 
Shaheen et al., \\
2011c
\end{tabular} & $\begin{array}{l}\text { Vegetation classification, } \\
\text { diversity \& distribution }\end{array}$ & $\checkmark$ & $\times$ & $\checkmark$ & $x$ & $x$ & CCA & Contribution of edaphic variations is missing \\
\hline 5 & \begin{tabular}{|c|} 
Siddiqui et al., \\
2011 \\
\end{tabular} & $\begin{array}{c}\text { Vegetation classification \& } \\
\text { description }\end{array}$ & $\checkmark$ & $\times$ & $x$ & $\times$ & $x$ & $x$ & Environmental influence is missing \\
\hline 6 & $\begin{array}{c}\text { Shaheen et al., } \\
2012\end{array}$ & $\begin{array}{l}\text { Vegetation classification, } \\
\text { diversity \& regeneration }\end{array}$ & $\checkmark$ & $\times$ & $\checkmark$ & $\checkmark$ & $x$ & CCA & $\begin{array}{c}\text { Overall, CCA model was non-significant } \\
\text { possibly because all the } 13 \text { studied sites } \\
\text { instead of plots were treated as samples with } \\
6 \text { explanatory variables, } \& \text { therefore, CCA did } \\
\text { not remain as a constrained model }\end{array}$ \\
\hline 7 & $\begin{array}{c}\text { Malik and } \\
\text { Malik, } 2012\end{array}$ & $\begin{array}{l}\text { Vegetation classification } \\
\text { and diversity }\end{array}$ & $x$ & $\times$ & $\times$ & $\times$ & $\times$ & $\times$ & Environmental influence is missing \\
\hline 8 & \begin{tabular}{|c|} 
Siddiqui et al., \\
2013 \\
\end{tabular} & \begin{tabular}{|c|}
$\begin{array}{c}\text { Vegetation classification \& } \\
\text { description }\end{array}$ \\
\end{tabular} & $\checkmark$ & $\times$ & $\times$ & $x$ & $x$ & $\times$ & Environmental influence is missing \\
\hline 9 & $\begin{array}{l}\text { Sher et al., } \\
2013\end{array}$ & \begin{tabular}{|c|}
$\begin{array}{c}\text { Vegetation classification } \& \\
\text { description }\end{array}$ \\
\end{tabular} & $x$ & $\times$ & $\times$ & $x$ & $x$ & $\times$ & Environmental influence is missing \\
\hline 10 & $\begin{array}{l}\text { Siddiqui et al., } \\
2014\end{array}$ & $\begin{array}{c}\text { Vegetation classification \& } \\
\text { contribution of topography } \\
\& \text { soil }\end{array}$ & $\checkmark$ & $\checkmark$ & $\times$ & $\checkmark$ & $\times$ & PCA & $\begin{array}{c}\text { A linear un-constrained multivariate model } \\
\text { (PCA) with studied environment as } \\
\text { supplementary variables is used. Therefore, } \\
\text { the question that how environment variations } \\
\text { are influencing species distribution is still un- } \\
\text { answered }\end{array}$ \\
\hline 11 & $\begin{array}{l}\text { Malik and } \\
\text { Malik, } 2014\end{array}$ & $\begin{array}{l}\text { Vegetation and species } \\
\text { phenology }\end{array}$ & $\times$ & $\times$ & $\times$ & $\times$ & $\times$ & $x$ & Environmental influence is missing \\
\hline 12 & \begin{tabular}{|c|} 
Shaheen et al., \\
2016
\end{tabular} & $\begin{array}{l}\text { Vegetation attributes and } \\
\text { fuelwood pressure }\end{array}$ & $x$ & $\times$ & $\times$ & $\checkmark$ & $x$ & PCA & Environmental influence is missing \\
\hline 13 & \begin{tabular}{|c|} 
Shaheen et al., \\
2017 \\
\end{tabular} & \begin{tabular}{|c|} 
Regeneration pattern of the \\
tree species
\end{tabular} & $x$ & $\times$ & $\times$ & $\checkmark$ & $\times$ & PCA & Environmental influence is missing \\
\hline 14 & \begin{tabular}{|c|} 
Shaheen et al., \\
2018 \\
\end{tabular} & \begin{tabular}{|c|} 
Vegetation \& \\
anthropogenic disturbance
\end{tabular} & $x$ & $\times$ & $\checkmark$ & $\checkmark$ & $x$ & PCA & Environmental influence is missing \\
\hline 15 & This study & $\begin{array}{l}\text { Vegetation classification, } \\
\text { indicators significance, } \\
\text { variation partitioning, } \\
\text { diversity \& distribution }\end{array}$ & $\checkmark$ & $\checkmark$ & $\checkmark$ & $\checkmark$ & $\checkmark$ & $\begin{array}{l}\text { DCA } \\
\& \\
\text { CCA }\end{array}$ & $\begin{array}{l}\text { Attempted to fill the gaps. Additionally, } \\
\text { detection of ecologically meaningful } \\
\text { vegetation types, MRPP, and variation } \\
\text { partitioning were also performed. Ordination } \\
\text { models were selected on the basis of gradient } \\
\text { length in the response data }\end{array}$ \\
\hline
\end{tabular}

T: topography; S: Soil; D: disturbance; HC: hierarchical classification; ISA: indicator species analysis; ORD: ordination $\times:$ No; $\sqrt{ }:$ Yes

The study area is diverse and the mean Shannon's diversity values of the four plant communities varied from 2.47-2.87, Simpson's diversity index from 0.89-0.94, reciprocal Simpson's diversity from 8.44-14.47, Pielou's evenness from 0.88-0.95, and Margalef's richness from 2.87-5.05. These values are comparable with the earlier work of Shaheen et al. (2011c), Malik and Malik (2012) and Sher et al. (2013) conducted in the same area. These diversity variations are also comparable with the other vegetation surveys in the Himalayas (Roy and Behera, 2005; Kharkwal, 2009). The $\beta$-diversity (amongst studied samples) in the study area varied from 47.88-78.65. It was observed 
that the number of species, indicator and significant indicator species of groups continuously decreased along the increasing elevation, whereas diversity values also showed the similar pattern except it again started to increase at the Ganga summit. However, the proportion of significant indicator species to indicators continuously increased along the elevation gradient, therefore depict the uniqueness of sub-alpine to alpine microhabitats in the Himalayas. The results of this study also suggested that the forests edges of the Sudhan Gali are more diverse than the forest interiors, and the similar results are also reported by Khan et al. (2019).

The role of topography and especially altitude is well documented (Shaheen et al., 2011c; Khan et al., 2019) in the Himalayas, however, CCA net effect testing proved that anthropogenic influences has come very close to topography and taken over the edaphic contribution in this regard. The order of importance of the studied environment is presented in Table 5 and Figure 7. All these disturbances and the rarest plant species are presented in Figure 8. Therefore, the study area need development and implementation of management plans for the restoration of important and valuable forest resources in this part of the western Himalaya.

\section{Conclusion and recommendations}

The species composition and vegetation structure of the observed four vegetation groups of the study area was significantly different. Therefore, these groups are supporting some rarest plant species and unique composition. The topography is detected as the leading driver of species distribution pattern variation followed by grazing and deforestation pressures. The highest correlation was observed between deforestation and thick coniferous forest patches, and grazing intensity and temperate grassland pastures in the area. Therefore, this study concluded that vegetation composition of the sub-alpine to alpine areas is changing at faster rates than the low elevation areas. The results of CCA (net effect) depicted that all the studied environmental variables (thirteen in total) were significantly important, and explains $73.1 \%$ variations. This study also concluded that the selected explanatory variables were determinants of the response (species) data variations and there was no single dominant environmental gradient in this part of the western Himalaya. Similarly, the highest $\beta$ diversity value of west Himalayan alpine scrubland association as compared to others, is representative of variety of microhabitats with unique flora. This also mean that even a minor variation in environment affects the high altitude vegetation more rapidly than the vegetation of low elevation areas.

An effective management plan for the restoration of vegetation in area are required including provision of fuel alternatives for the local masses. This study recommend that the importance of local biodiversity and consequences of its depletion should be regularly addressed and communicated to local masses. The launching of afforestation and reforestation programs, and their continuous monitoring will prove effective. Similarly, stimulus of medicinal species cultivation can help a lot in this regard. The provision of free seedlings of valuable native species, strict enforcement of biodiversity protection law, periodic monitoring of endangered species populations are some other effective tools. All this is necessary because these wild relatives can serve as an important germplasm for the future drugs, and different stress and disease tolerance characteristics which can be used for the improvement of masses health, and existing valuable agricultural species respectively. 

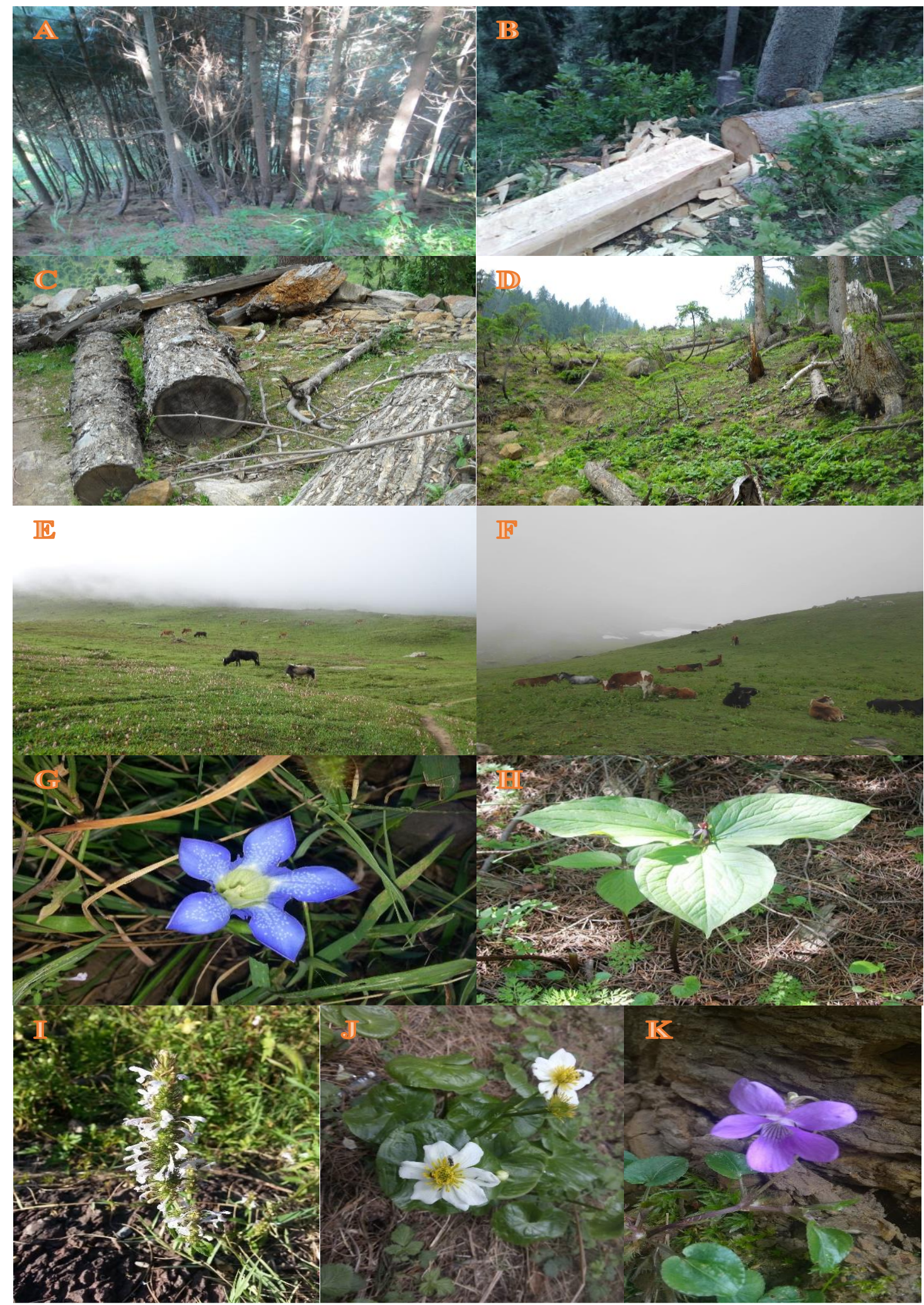

Figure 8. Some visuals depicting the influential anthropogenic factors (A: forest fire; B-D: deforestation; E-F: grazing), and extremely rare plant species (G: Gentiana kurroo; H:

Trillium govanianum; I: Nepeta podostachys; J: Caltha palustris var. alba; K: Viola odorata) of the western Himalayan forests of AJ\&K, Pakistan 
We concluded that overall vegetation of the area is under heavy anthropogenic pressure and sustainable use of these natural resources is recommended. Various concerned departments like wildlife, forest, environment, tourisms and local government should play their role in launching of awareness campaigns, conservation/management plan development, execution and implementation to save this wealth of the area.

This study observed that over-exploitation of medicinal plant species especially from the Ganga summit and adjacent alpine area is resulted in least population size of Geum urbanum, Gentiana kurroo, Nepeta podostachys, Caltha palustris var. alba, Viola odorata and Trillium govanianum. According to literature, species like Saussurea costus and Rheum australe were once surviving and now become extinct in the area. Therefore, future studies like documentation of rate of population fluctuation, potential habitat mapping under present, past and future climate scenarios should be conducted immediately to ensure their sustainable use, forest management and conservation.

\section{REFERENCES}

[1] Abdel Khalik, K., Al-Gohary, I., Al-Sodany, Y. (2017): Floristic composition and vegetation: environmental relationships of Wadi Fatimah, Mecca, Saudi Arabia. - Arid Land Research and Management 31: 316-334.

[2] Akhlaq, R., Amjad, M. S., Qaseem, M. F., Fatima, S., Chaudhari, S. K., Khan, A. M., Khan, S., Malik, N. Z., Gardazi, S. M. H., Bibi, A., Sabaoon (2018): Species diversity and vegetation structure from different climatic zones of Tehsil Harighel, Bagh, Azad Kasmir, Pakistan analysed through multivariate techniques. - Applied Ecology and Environmental Research 16(4): 5193-5211.

[3] Ali, S. I., Qaiser, M. (1995-2009): Flora of West Pakistan. - Department of Botany, University of Karachi, Karachi, Pakistan.

[4] Anonymous. (2015): AJK at Glance. - http://pndajk.gov.pk (accessed: 4/2/2017).

[5] Bürzle, B., Schickhoff, U., Schwab, N., Oldeland, J., Müller, M., Böhner, J., Chaudhary, R. P., Scholten, T., Dickoré, W. B. (2017): Phytosociology and ecology of treeline ecotone vegetation in Rolwaling Himal, Nepal. - Phytocoenologia 47: 197-220.

[6] Champion, S. H., Seth, S. K., Khattak, G. M. (1965): Forest Types of Pakistan. - Pakistan Forest Institute, Peshawar.

[7] Chandra, S., Singh, A., Singh, C. P., Nautiyal, M. C., Rawat, L. S. (2018): Vascular plants distribution in relation to topography and environmental variables in alpine zone of Kedarnath Wild Life Sanctuary, West Himalaya. - Journal of Mountain Science 15: 1936-1949.

[8] Cox, G. W. (1967): Laboratory Manual of General Ecology. - William C Brown, Dubuque.

[9] Curtis, J. T., McIntosh, R. P. (1950): The interrelations of certain analytic and synthetic phytosociological characters. - Ecology 31: 434-455.

[10] Devetter, M., Háněl, L., Řeháková, K., Doležal, J. (2017): Diversity and feeding strategies of soil microfauna along elevation gradients in Himalayan cold deserts. - PloS One 12: e0187646. https://doi.org/10.1371/journal.pone.0187646.

[11] Dufrêne, M., Legendre, P. (1997): Species assemblages and indicator species: the need for a flexible asymmetrical approach. - Ecol. Monogr. 67(3): 345-366.

[12] EFLORAS (2012): Flora of Pakistan @ eflorasrg. http://www.efloras.org/flora_page.aspx?flora_id=5 (accessed 16/9/2018).

[13] Haq, F. (2018): Vegetation mapping above tree line in Nandiar Valley, western Himalayas. A multivariate approach. - Acta Ecologica Sinica 38: 15-20. 
[14] Ilyas, M., Qureshi, R., Akhtar, N., Ziaul-Haq, Khan, A. M. (2018): Floristic diversity and vegetation structure of the remnant subtropical broad leaved forests from Kabal Valley, Swat, Pakistan. - Pak. J. Bot. 50(1): 217-230.

[15] Kamran, S., Khan, S. M., Ahmad, Z., Rahman, A. U., Iqbal, M., Manan, F., Haq, Z. U., Ullah, S. (2019): The role of graveyards in species conservation and beta diversity: a vegetation appraisal of sacred habitats from Bannu, Pakistan. - Journal of Forestry Research. https://doi.org/10.1007/s11676-019-00893-1.

[16] Khan, A. M. (2001): Phytosociology, floristic composition \& phenological studies with special reference to rangeland at Sudhan Gali and Ganga Chotti hills (District Bagh AK) during, 2000. - MSc. Thesis (unpublished), Department of Botany, AJ\&K University, Muzaffarabad, Pakistan.

[17] Khan, A. M., Qureshi, R., Qaseem, M. F., Munir, M., Ilyas, M., Saqib, Z. (2015): Floristic checklist of district Kotli, Azad Jammu \& Kashmir. - Pak. J. Bot. 47(5): 19571968.

[18] Khan, A. M., Qureshi, R., Qaseem, M. F., Ahmad, W., Saqib, Z., Habib, T. (2016): Status of basic taxonomic skills in botanical articles related to Azad Jammu and Kashmir, Pakistan: A Review. - J. Bioresource Manage. 3(3): 22-54.

[19] Khan, A. M., Qureshi, R., Arshad, M., Mirza, S. N. (2018a): Climatic and flowering phenological relationships of Western Himalayan Flora of Muzaffarabad District, Azad Jammu and Kashmir, Pakistan. - Pak. J. Bot. 50(3): 1093-1112.

[20] Khan, A. M., Qureshi, R., Saqib, Z., Habib, T., Ilyas, M., Maqsood, M., Kosar, R., Akram, M., Rahim, B. Z. (2018b): A novel study of the interrelationship of seasonality, satellite data and weed compositional changes of the agro-ecological system of Gujrat, Pakistan. - Applied Ecology and Environmental Research 16(3): 2995-3018. http://dx.doi.org/10.15666/aeer/1603_29953018.

[21] Khan, A. M., Qureshi, R., Saqib, Z. (2019): Multivariate analyses of the vegetation of the western Himalayan forests of Muzaffarabad district, Azad Jammu and Kashmir, Pakistan. - Ecological Indicators 104: 723-736. https://doi.org/10.1016/j.ecolind.2019.05.048.

[22] Kharkwal, G. (2009): Qualitative analysis of tree species in evergreen forests of Kumaun Himalaya, Uttarakhand, India. - African Journal of Plant Science 3(3): 49-52.

[23] Legendre, P., Legendre, L. (1998): Numerical ecology. $2^{\text {nd }}$ ed. - Elsevier B., Amsterdam.

[24] Magurran, A. E. (1988): Ecological Diversity and Its Measurement. Chap. 1: Why Diversity? - Springer, Dordrecht, pp. 1-5.

[25] Malik, Z. H., Malik, N. Z. (2012): High altitude forest composition diversity and its component in a part of Ganga chotti and Bedori hills district Bagh. Azad Jammu and Kashmir, Pakistan. - Acta Geographica Debrecina Landscape \& Environment 6(1): 3140.

[26] Malik, Z. H., Malik, N. Z. (2014): Phenological patterns among the vegetation of Ganga Chotti and Bedori Hills in a moist temperate to alpine forests. - International Journal of Biodiversity and Conservation 6(6): 444-451.

[27] Malik, Z. H., Hussain, F., Malik, N. Z. (2007): Life form and leaf size spectra of plant communities Harbouring Ganga Chotti and Bedori Hills during 1999-2000. - Int. J. Agric. Biol. 9(6): 833-838.

[28] Margalef, R. (1958): Temporal Succession and Spatial Heterogeneity in Phytoplankton. In: Buzzati-Traverso, A. A. (ed.) Perspectives in Marine Biology. University of California Press, Berkeley, CA.

[29] Nakaike, T., Malik, S. (1992): Cryptogamic Flora of Pakistan. - National Science Museum, Tokyo.

[30] Nasir, E., Ali, S. I. (1971-1995): Flora of West Pakistan. - Department of Botany, University of Karachi, Karachi.

[31] Nelson, D. W., Sommer, L. E. (1982): Total Carbon, Organic Carbon, and Organic Matter 1. - In: Page, A. L. (ed.) Methods of Soil Analysis. Part 2. Agronomy Series No. 9. ASA, Madison, WI, pp. 539-579. 
[32] Olsen, S. R., Cole, C. V., Watanabe, F. S., Dean, L. A. (1954): Estimation of available phosphorus in soils by extraction with sodium bicarbonate. - USDA Circ. 939: 1-19.

[33] Othman, F., Chowdhury, M. S. U., Sakai, N., Shaaban, M. G., Shimizu, Y. (2014): Identification of pollution loading in a tropical river basin: a case study of Selangor River, Malaysia. - WIT Trans Built Environ 156. DOI: 10.2495/ESBE140121.

[34] Pielou, E. C. (1975): Ecological Diversity. - Wiley, New York.

[35] PMD (2018): Pakistan Meteorological Department. - National Agromet Centre, Islamabad, Pakistan.

[36] Qamer, F., Shehzad, K., Abbas, S., Murthy, M. S. R., Xi, C., Gilani, H., Bajracharya, B. (2016): Mapping deforestation and forest degradation patterns in western Himalaya, Pakistan. - Remote Sensing 8(5): 385.

[37] Rahman, I. U., Khan, N., Ali, K. (2017): Classification and ordination of understory vegetation using multivariate techniques in the Pinus wallichiana forests of Swat Valley, northern Pakistan. - The Science of Nature 104: 24. DOI: 10.1007/s00114-017-1431-2.

[38] Richards, L. A. (1954): Diagnosis and Improvement of Saline and Alkali Soils. - In: Richards, L. A. (ed.) Agriculture Handbook No. 60. United States Salinity Laboratory, Riverside, CA.

[39] Roy, P. S., Behera, M. D. (2005): Assessment of biological richness in different altitudinal zones in the Eastern Himalayas, Arunachal Pradesh, India. - Curr. Sci. India 88(2): 321-330.

[40] Saeeda, S., Zakir, S. (2012): Environmental awareness as a tool to promote the wise practices of wild plant resources in the Himalayas (Sudhangali-Kashmir, Pakistan). PUTAJ Sciences 19: 139-154.

[41] Saima, S., Altaf, A., Faiz, M. H., Shahnaz, F., Wu, G. (2018): Vegetation patterns and composition of mixed coniferous forests along an altitudinal gradient in the Western Himalayas of Pakistan. - Austrian Journal of Forest Science 135: 159-180.

[42] Salama, F., El-Ghani, M. A., Gadallah, M., El-Naggar, S., Amro, A. (2016): Characteristics of desert vegetation along four transects in the arid environment of southern Egypt. - Turkish Journal of Botany 40: 59-73.

[43] Salama, F., El-Ghani, M. A., Gadalla, M., Ramadan, T., Galal, H., Gaafar, A. (2018): Vegetation patterns and floristic composition along elevation gradient on Jabal Musa, South Sinai, Egypt. - Catrina-The International Journal of Environmental Sciences 16: 41-57.

[44] Schofield, R. K., Taylor, A. W. (1955): The measurement of soil pH. - Soil Sci. Soc. Am. J. 19: 164-167.

[45] Shaheen, H., Qureshi, R. A., Shinwari, Z. K. (2011a): Structural diversity, vegetation dynamics and anthropogenic impact on lesser Himalayan sub-tropical forests of Bagh district, Kashmir. - Pak. J. Bot. 43(4): 1861-1866.

[46] Shaheen, H., Qureshi, R. A., Ullah, Z., Ahmad, T. (2011b): Anthropogenic pressure on the western Himalayan moist temperate forests of Bagh, Azad Jammu \& Kashmir. - Pak. J. Bot. 43(1): 695-703.

[47] Shaheen, H., Khan, S. M., Harper, D. M., Ullah, Z., Qureshi, R. A. (2011c): Species diversity, community structure, and distribution patterns in western Himalayan alpine pastures of Kashmir, Pakistan. - Mt. Res. Dev. 31(2): 153-159.

[48] Shaheen, H., Ullah, Z., Khan, S. M., Harper, D. M. (2012): Species composition and community structure of western Himalayan moist temperate forests in Kashmir. - Forest Ecology and Management 278: 138-145.

[49] Shaheen, H., Azad, B., Mushtaq, A., Khan, R. W. A. (2016): Fuelwood consumption pattern and its impact on forest structure in Kashmir Himalayas. - Bosque 37(2): 419424.

[50] Shaheen, H., Nasar, S., Aziz, S. Mumtaz, N., Aziz, S. (2017): Regeneration pattern in subtropical and moist temperate forest stands of Kashmir Himalayas. - Environment and Ecology Research 5(5): 340-346. DOI: 10.13189/eer.2017.050503. 
[51] Shaheen, H., Riffat, A., Salika, M., Firdous, S. S. (2018): Impacts of roads and trails on floral diversity and structure of Ganga-Choti forest in Kashmir Himalayas. - Bosque 39(1): 71-79.

[52] Shannon, C. E., Weaver, W. (1949): The Mathematical Theory of Communication. University of Illinois Press, Urbana, IL.

[53] Sher, Z., Hussain, F., Badshah, L. (2013): Phytosociology of summer vegetation of Sudan Galli Hills, District Bagh, Azad Kashmir, Pakistan. - Pak. J. Bot. 45(1): 1-9.

[54] Siddiqui, M. F., Ahmed, M. Khan, N., Khan, I. A. (2010a): A quantitative description of moist temperate conifer forests of Himalayan region of Pakistan and Azad Kashmir. International Journal of Biology and Biotechnology 7(3): 175-185.

[55] Siddiqui, M. F., Ahmed, M., Shaukat, S. S., Khan, N. (2010b): Advanced multivariate techniques to investigate vegetation-environmental complex of pine forests of moist temperate areas of Pakistan. - Pak. J. Bot. 42(SI): 267-293.

[56] Siddiqui, M. F., Ahmed, M., Hussain, S. S., Shaukat, S. S., Khan, N. (2011): Vegetation description and current status of moist temperate coniferous forests of Himalayan and Hindukush region of Pakistan. - FUUAST J. Biol. 1(2): 99-114.

[57] Siddiqui, M. F., Shaukat, S. S., Ahmed, M., Khan, N., Khan, I. A. (2013): VegetationEnvironment relationship of conifer dominating forests of moist temperate belt of Himalayan and Hindukush regions of Pakistan. - Pak. J. Bot. 45(2): 577-592.

[58] Siddiqui, M. F., Shaukat, S. S., Ahmed, M. (2014): Topographic and edaphic control of arboreal vegetation and the distribution and growth of tree species in moist temperate areas of Himalayan and Hindukush regions of Pakistan. - Pakistan J. Bot. 46(4): 11871196.

[59] Stewart, R. R. (1972): An Annotated Catalogue of the Vascular Plants of West Pakistan and Kashmir. - Fakhri Printing Press, Karachi.

[60] Ter Braak, C. J. F. (1986): Canonical correspondence analysis: a new eigenvector technique for multivariate direct gradient analysis. - Ecology 67(5): 1167-1179.

[61] Ter Braak, C. J. F., Smilauer, P. (2012): Canoco 5. Windows Release (5.00). Software for Canonical Community Ordination. - Microcomputer Power, Ithaca.

[62] Tewari, V. P., Verma, R. Kn Gadow, K. (2017): Climate change effects in the Western Himalayan ecosystems of India: evidence and strategies. - Forest Ecosystems 4(1): 13.

[63] TROPICOS (2012): Flora of Pakistan. http://www.tropicos.org/NameSearch.aspx?projectid=32 (accessed 22/9/2018).

[64] Waseem, M., Shah, M. A. U., Qureshi, R. A., Muhammad, I., Afza, R., Yousaf, S. (2006): Ethnopharmacological survey of plants used for the treatment of stomach, diabetes, and ophthalmic diseases in Sudhan Gali, Kashmir, Pakistan. - Acta Botanica Yunnanica 28(5): 535-542.

[65] Zakaria, R., Nizam, M. S., Faridah-Hanum, I. (2019): Association of tree communities with soil properties in a semi deciduous forest of Perlis, Peninsular Malaysia. - Biotropia - The Southeast Asian Journal of Tropical Biology 27: 8. DOI: 10.11598/btb.2020.27.1.1122. 